\title{
EXISTENCE OF TRAVELING WAVES IN A BIODEGRADATION MODEL FOR ORGANIC CONTAMINANTS*
}

\author{
REGAN MURRAY ${ }^{\dagger}$ AND J. X. XIN ${ }^{\dagger}$
}

\begin{abstract}
We study a biodegradation model for the time evolution of concentrations of contaminant, nutrient, and bacteria. The bacteria has a natural concentration which will increase when the nutrient reaches the substrate (contaminant). The growth utilizes nutrients and degrades the substrate. Eventually, such a process removes all the substrate and can be described by traveling wave solutions. The model consists of advection-reaction-diffusion equations for the substrate and nutrient concentrations and a rate equation (ODE) for the bacteria concentration. We first show the existence of approximate traveling wave solutions to an elliptically regularized system posed on a finite domain using degree theory and the elliptic maximum principle. To prove that the approximate solutions do not converge to trivial solutions, we construct comparison functions for each component and employ integral identities of the governing equations. This way, we derive a priori estimates of solutions independent of the length of the finite domain and the regularization parameter. The integral identities take advantage of the forms of coupling in the system and help us obtain optimal bounds on the traveling wave speed. We then extend the domain to the infinite line limit, remove the regularization, and construct a traveling wave solution for the original set of equations satisfying the prescribed boundary conditions at spatial infinities. The contaminant and nutrient profiles of the traveling waves are strictly monotone functions, while the biomass profile has a pulse shape.
\end{abstract}

Key words. biodegradation, organic contaminants, traveling waves

AMS subject classifications. 92C05, 92C45, 34B15, 35J25

PII. S0036141096313392

1. Introduction. Thousands of chemical spills contaminate subsurface aquifers used for drinking water and agriculture in the United States. A promising technology for cleaning up subsurface organic contamination is in-situ bioremediation. This technique works by stimulating the bacteria already present in the soil to use the contaminant as a source of food, thereby transforming it into nontoxic components such as carbon dioxide and water. Among a variety of restoration technologies, insitu bioremediation has been shown to be the most economical for remediating certain contaminants. Several experiments have shown that this method takes much less time and is less costly than the traditional pumping and filtering techniques. For example, one site was estimated to take 100 years to clean up using the pump and treat method; in-situ bioremediation took only 10 months [3]. Moreover, while many methods simply remove the contaminant from the site and thereby create a disposal problem, in-situ bioremediation has the potential to completely transform organic contaminants into neutral compounds by utilizing the indigenous bacteria [6].

In-situ bioremediation involves a complex combination of biological and chemical properties and fluid dynamics rendering scientific predictions difficult. Mathematical models are particularly useful in understanding the interplay between the various mechanisms in addition to making predictions, analyzing the significance of physical

* Received by the editors December 9, 1996; accepted for publication (in revised form) February 10, 1998; published electronically September 29, 1998.

http://www.siam.org/journals/sima/30-1/31339.html

${ }^{\dagger}$ Program in Applied Mathematics, Department of Mathematics, University of Arizona, Tucson, AZ 85721 (rmurray@math.arizona.edu, xin@math.arizona.edu). The research of the first author was partially supported by a graduate teaching assistantship at the Program in Applied Mathematics, University of Arizona, and by the Center of Nonlinear Studies, Los Alamos National Laboratory. The research of the second author was supported by NSF grants DMS-9302830 and DMS-9625680. 
parameters, and providing theoretical interpretations of experiments. Several models for in-situ bioremediation have been presented in the literature; see [2], [5]. In this paper, we consider the model presented in [7] and [8], for it is the simplest model to capture the fundamental aspects of degradation.

The biodegradation model is

$$
\begin{aligned}
& R_{f} S_{t}+\left(v S-D S_{x}\right)_{x}=-R_{s}, \quad x \in R^{1}, \\
& A_{t}+\left(v A-D A_{x}\right)_{x}=-\gamma R_{s}, \\
& M_{t}=Y R_{s}-b\left(M-M_{0}\right)
\end{aligned}
$$

where $R_{f}, b, M_{0}, \gamma, v, D, Y$ are all positive constants. The subscripts $t$ and $x$ denote time and space derivatives, while the subscripts $s$ and $f$ are used to distinguish between the reaction term $R_{s}$ and retardation factor $R_{f}$. The variables are $(S, A, M)$ where $S$ is the aqueous phase concentration of the electron donor (substrate), $A$ is the electron acceptor (nutrient concentration), and $M$ is the biomass concentration. The constant $M_{0}$ is the neutral background biomass concentration. $R_{f}>1$ is the retardation factor of the electron donor (substrate) $S$ due to sorption. The parameter $b$ is the cell decay coefficient for the bacteria population $M . \gamma$ is a coefficient equal to the mass of $A$ utilized by the bacteria per unit mass of $S$ degraded. $D$ is the diffusion coefficient and $Y$ is the yield coefficient (mass of bacterial cells produced per mass of $S$ degraded). The parameter $v$ is the constant pore water velocity and $M_{0}$ is the background biomass concentration.

The biodegradation rate, $R_{s}$, is given by the Monod kinetic model

$$
R_{s}=\frac{q M S A}{\left(K_{S}+S\right)\left(K_{A}+A\right)},
$$

where $q$ is the maximum specific rate of substrate utilization, and $K_{S}, K_{A}$ are the half-maximum rate concentrations of $S$ and $A$. Note that the system assumes linear sorption of the electron donor (represented by the term $R_{f}$ ), no sorption for the electron acceptor, and a constant minimal background bacteria population $M_{0}$ (as an equilibrium between cell growth and decay). In this model, microbes are attached to the soil particles and only consume aqueous phase species.

The system (1.1)-(1.3) is one of the simplest systems in the literature; however, it contains very rich phenomena. Oya and Valocchi [8] and Valocchi [10] recently studied traveling waves in this system. They observed that "bioavailability" of the chemical constituents is an important requirement for successful biodegradation. Since the microbes are assumed to be attached to solids [5], the dissolved contaminant and nutrients must be transported to the bacteria. This is represented in the above system by the water velocity $v$ and the sorption effect $R_{f}>1$. When the nutrients reach the biomass, a biologically active zone forms and propagates. This zone supports respiration, that is, nutrient consumption, contaminant degradation, and an increase in the microbial population. [8] is the first paper to propose the idea that the biologically active zone can be mathematically modeled as traveling waves. The retreating dissolved solute concentration and the advancing nutrient concentration move together as traveling fronts, while the bacteria concentration tags along as a traveling pulse.

In this paper, we establish the existence of traveling waves in (1.1)-(1.3) under the sole condition that $R_{f}>1$. The traveling wave solutions are of the form

$$
S=S(x-c t), A=A(x-c t), M=M(x-c t),
$$


where $(S, A, M)$ as functions of $\xi \equiv x-c t$ satisfy the boundary conditions

$$
S(-\infty)=0, \quad S(+\infty)=S^{+}, A(-\infty)=A_{-}, \quad A(+\infty)=0, M( \pm \infty)=M_{0},
$$

and $A_{-}$and $S^{+}$are prescribed positive constants. Using (1.5), (1.1)-(1.3) are transformed into

$$
\begin{gathered}
D S_{\xi \xi}+\left(R_{f} c-v\right) S_{\xi}=R_{s}, \\
D A_{\xi \xi}+(c-v) A_{\xi}=\gamma R_{s}, \\
c M_{\xi}=-Y R_{s}+b\left(M-M_{0}\right),
\end{gathered}
$$

along with the boundary conditions

$$
S(-\infty)=0, S(+\infty)=S^{+}, A(-\infty)=A_{-}, A(+\infty)=0, M( \pm \infty)=M_{0}
$$

To prove the existence of a solution to (1.7)-(1.9), we follow a framework similar to that of Berestyki, Nicolaenko, and Sheurer [1] and Xin [11], and consider the system on a bounded domain $[-d, d], d>0$. We define a fixed point map such that any solution to the fixed point problem is also a solution of (1.7)-(1.9) on the bounded domain. By determining the Leray-Schauder degree of the map (see [13] for details), we show that a solution exists to the fixed point problem. Finally, we extend the solution to the entire real line. This is a well-known technique.

It is important to note that equations (1.1)-(1.3) are substantially different than the two-equation, reaction-diffusion, combustion system studied in [1] and require new ingredients for the proof of existence. In the biodegradation model, the fronts propagate as a result of distinct advective velocities rather than reaction-diffusion. We believe traveling wave fronts in advection systems have not been much explored. In this case, although the method of [1] applies, many new steps are needed. For instance, finding good bounds on the wave speed requires a substantial amount of work on the advection and reaction terms. In addition, the two advection equations cannot be manipulated to define pointwise inequalities as in [1]. Instead, we develop integral inequalities based on the conserved quantity $\gamma R_{f} S-A$ (see Lemma 3.4).

Many other differences between the biodegradation system and [1] are noteworthy. First, while the fronts in [1] are strictly monotone, the biomass profile in (1.1)-(1.3) forms a pulse. Rather than following directly from the maximum principle, new arguments are necessary to estimate the maximum of $M$ (see Proposition 2.1). Second, the condition to fix translation invariance, $A(0)=\theta$, is not a priori optimal as it is in [1], where $\theta$ is the ignition temperature. In this paper, the value of $\theta$ is updated three times to prevent the solutions of (1.1)-(1.3) from converging to trivial solutions in the limit $d \rightarrow \infty$ (see Proposition 3.1 and Lemmas 3.3 and 3.4). In particular, much work is required to show that $S$ does not tend to zero as the domain extends to the real line. Finally, we develop integral identities to show that the boundary conditions remain as $d \rightarrow \infty$; see Theorem 4.1 and Lemma 5.1. Using integral identities instead of pointwise estimates to control wave speeds and large space asymptotic behavior of solutions is an efficient way of handling systems with more than two equations.

The main result of the paper is the following.

THEOREM 1.1 (existence of traveling waves). Under the condition that the retardation factor $R_{f}>1$, the system (1.7)-(1.9) with $R_{s}$ given by (1.4) admits a classical traveling wave solution $(S, A, M, c)$ of the form (1.5) satisfying the boundary conditions in (1.10). Moreover,

$$
0<S(\xi)<S^{+}, S^{\prime}(\xi)>0,0<A(\xi)<A_{-}, A^{\prime}(\xi)<0 \quad \forall \xi \in R^{1},
$$




$$
\begin{aligned}
& c=\frac{v\left(A_{-}+\gamma S^{+}\right)}{A_{-}+\gamma R_{f} S^{+}}, \\
& M_{0}<M \leq M_{0}+Y \frac{\left(R_{f}-1\right) A_{-} S^{+}}{A_{-}+\gamma S^{+}} \quad \forall \xi \in R^{1} .
\end{aligned}
$$

We see from Theorem 1.1 that the profiles of substrate and nutrient concentrations $(S$ and $A$ ) are strictly monotone functions of $\xi$ and that the wave speed $c$ is independent of the parameters $Y, b$ of the $M$ equation. The explicit formula for the wave speed $c$ in terms of the left and right states of solutions is reminiscent of the Rankine-Hugoniot formula for viscous shock waves in conservative equations; see [12] for such traveling waves arising in solute transport problems. The maximum norm bound of the biomass $M$, on the other hand, depends on the parameters and boundary conditions of the $(S, A)$ equations as well as the yield constant $Y$ of the $M$ equation. Numerical simulations in [8] and those of the present authors show that $M$ forms an asymmetric pulse with one maximum. The condition $R_{f}>1$ physically means that the advective velocity of $S$ is slower than that of $A$; hence, the two concentrations mix, which is essential for the biomass to grow and the three components to travel together. As pointed out in [8], there are no traveling waves if $R_{f}=1$. In fact, it is obvious from (1.13) that $M \equiv M_{0}$ if $R_{f}=1$, and no traveling pulse can form in $M$. It remains an interesting problem to find out if the traveling waves are unique up to a constant translation in $\xi$ and if $M$ always achieves one maximum.

The main result is significant, for it shows that there is a simple, well-understood solution to a very complicated phenomena. This solution occurs under a particular set of parameters; however, numerical studies suggest that this is the most common solution. In [8], the authors find waves with oscillating front shapes in time; this proof will be left for a future paper. Finally, many of the important factors required for the implementation of in-situ bioremediation are determined in Theorem 1.1, such as the speed of the traveling fronts and the maximum and minimum concentrations of the biomass.

The second result of the paper is the following.

TheOREM 1.2 (existence and uniqueness of traveling waves). The system (1.7)(1.9) with $D=0$ and $R_{s}$ given by (1.4) admits a unique, classical traveling wave solution $(S, A, M, c)$ of the form (1.5) satisfying the boundary conditions in (1.10) and the bounds given in Theorem 1.1.

Theorem 1.2 is proven in section 6 . By eliminating the diffusion term $(D=0)$, the system of equations (1.7)-(1.9) can be reduced to a set of three, first-order ordinary differential equations. A conserved quantity enables us to reduce the system further to two equations. Thus, a phase portrait solution is easily obtained and we show that the traveling wave solution is unique. It is interesting to note that the question of existence and uniqueness for the system with diffusion can also be considered by examining the flow in the phase plane. In this case, the phase space has five dimensions although it is reduced to four via the conserved quantity. One of the equilibrium points is degenerate so it is difficult to determine the flow path. As such, we have elected to pursue the proof of existence by utilizing degree theory. Moreover, numerical studies [8] suggest that the traveling wave solution with diffusion is not unique.

The rest of the paper is organized as follows. In section 2, we show the existence of solutions to a regularized system on any finite interval via degree theory, based on maximum principles and preliminary a priori bounds. In section 3, we carry out refined a priori estimates of solutions as the length of the interval tends to infinity. In 
particular, we obtain uniform estimates of the wave speed and bound it strictly inside the interval $\left(\frac{v}{R_{f}}, v\right)$. In section 4 , utilizing the estimates of section 3 , we pass to the infinite line limit of solutions and justify the validity of the boundary conditions of the limiting solutions. In section 5, we obtain further $\varepsilon$-independent bounds of solutions, pass to the $\varepsilon \rightarrow 0$ limit of solutions, and finish the proof of Theorem 1.1. In section 6 , we prove Theorem 1.2.

2. A regularized system on finite intervals. In this section, we construct solutions to a regularized system with Dirichlet boundary conditions. In the following sections, these solutions are shown to converge to the desired traveling wave solutions of (1.7)-(1.9) as we pass to the infinite line limit and remove the regularization. We add an elliptic regularization term $\epsilon M_{\xi \xi}$ to the left side of $(1.9)$ with $\epsilon \in(0,1)$ so that the existence problem is turned into a fixed point problem for which classical LeraySchauder degree theory [4], [13] is available. We derive a priori bounds of solutions and compute the degree using its homotopic invariance as in [1] and [11]. The nonzero degree (equal to -1 in our case) implies the existence of a solution.

Let us first normalize the original system (1.7)-(1.9) so that $K_{S}$ and $K_{A}$ are scaled to one. Define $S=K_{S} \hat{S}, A=K_{A} \hat{A}, q=K_{S} \hat{q}, \gamma=\frac{K_{A}}{K_{S}} \hat{\gamma}$, and $Y=\frac{1}{K_{S}} \hat{Y}$. Then (1.7)-(1.9) remains the same under hat variables and parameters except that $K_{S}$ and $K_{A}$ are replaced by one. With no loss of generality, we also set $\hat{q}=1$. The normalized system (without the hats) reads

$$
\begin{gathered}
D S_{\xi \xi}+\left(R_{f} c-v\right) S_{\xi}=M S A(1+S)^{-1}(1+A)^{-1} \\
D A_{\xi \xi}+(c-v) A_{\xi}=\gamma M S A(1+S)^{-1}(1+A)^{-1} \\
c M_{\xi}=-Y M S A(1+S)^{-1}(1+A)^{-1}+b\left(M-M_{0}\right)
\end{gathered}
$$

under the boundary conditions (1.10).

We propose to study the following regularized elliptic system and the associated boundary value problem on a finite interval $[-d, d]$ :

$$
\begin{array}{r}
D S_{\xi \xi}+\left(R_{f} c-v\right) S_{\xi}=R_{s, \varepsilon}^{(1)}, \\
D A_{\xi \xi}+(c-v) A_{\xi}=\gamma R_{s, \varepsilon}^{(2)}, \\
\varepsilon M_{\xi \xi}+c M_{\xi}=-Y R_{s, \varepsilon}^{(3)}+b\left(M-M_{0}\right),
\end{array}
$$

with $\varepsilon \in(0,1)$ and the boundary conditions

$$
S(-d)=A(d)=0, S(d)=S^{+}, A(-d)=A_{-}, M( \pm d)=M_{0} .
$$

To remove the translation invariance of traveling wave solutions of an unknown speed $c$, we also impose the additional normalization condition

$$
A(0)=\theta, \quad \theta \in\left(0, A_{-}\right),
$$

with $\theta$ prescribed. The modified reaction terms $R_{s}^{(i)}, i=1,2,3$, are

$$
\begin{aligned}
R_{s, \varepsilon}^{(1)} & =\frac{|M| S|A|}{(1+\varepsilon|M|)(1+|S|)(1+|A|)}, \\
R_{s, \varepsilon}^{(2)} & =\frac{|M||S| A}{(1+\varepsilon|M|)(1+|S|)(1+|A|)}, \\
R_{s, \varepsilon}^{(3)} & =\frac{M|S||A|}{(1+\varepsilon|M|)(1+|A|)(1+|S|)} .
\end{aligned}
$$


Note that we modify the reaction terms by the factor $(1+\varepsilon|M|)$ which is crucial to obtain the first upper bound on $M$. The variables $S, A, M$, and $c$ depend on both $d$ and $\varepsilon$; however, to simplify the notation, we do not specify this dependence until it becomes necessary in the later sections.

In order to use degree theory, we consider a parametrized family of equations indexed by $\tau \in[0,1]$,

$$
\begin{array}{r}
D S_{\xi \xi}+\left(R_{f} c-v\right) S_{\xi}=\tau R_{s, \varepsilon}^{(1)}, \\
D A_{\xi \xi}+(c-v) A_{\xi}=\gamma \tau R_{s, \varepsilon}^{(2)}, \\
\varepsilon M_{\xi \xi}+c M_{\xi}=-Y \tau R_{s, \varepsilon}^{(3)}+b\left(M-M_{0}\right),
\end{array}
$$

under the boundary conditions (2.7) and the imposed condition (2.8).

Note that if $\tau=0,(2.10)-(2.12)$ under (2.7) and (2.8) has a unique solution. Without (2.8), the system is uniquely solvable for any given $c$, as a two-point boundary value problem for second-order ordinary differential equations. The solutions are

$$
\begin{aligned}
& S(\xi)=S^{+} \frac{1-e^{\left(-\frac{c R-v}{D}\right)(\xi+d)}}{1-e^{\left(-\frac{c R-v}{D}\right)(2 d)}}, \\
& A(\xi)=A_{-} \frac{1-e^{\left(-\frac{c-v}{D}\right)(\xi-d)}}{1-e^{\left(\frac{c-v}{D}\right)(2 d)}}, \\
& M(\xi)=M_{0} .
\end{aligned}
$$

The extra condition $A(0)=\theta$ implies

$$
\theta=A_{-} \frac{1-e^{d\left(\frac{c-v}{D}\right)}}{1-e^{2 d\left(\frac{c-v}{D}\right)}}=A_{-} \frac{1}{1+e^{d\left(\frac{c-v}{D}\right)}},
$$

which uniquely determines $c$, since the right-hand side is a monotone function of $c$ and ranges between zero and $A_{-}$.

Next we derive a priori estimates on solutions of (2.10)-(2.12) under the boundary and normalization conditions (2.7) and (2.8) independent of $\tau \in[0,1]$.

Proposition 2.1. Let $\tau \in[0,1]$ and let $(S, A, M, c)$ be a solution to (2.10)(2.12) subject to the boundary conditions (2.7). Then $\forall \tau \in[0,1]$ and $\forall \xi \in[-d, d]$, we have the following inequalities:

$$
\begin{gathered}
0 \leq S(\xi) \leq S^{+}, \quad 0 \leq A(\xi) \leq A_{-} \\
M_{0} \leq M(\xi) \leq \\
\frac{1}{2 \varepsilon}\left(\varepsilon M_{0}+\frac{Y}{b}-1+\sqrt{\left(1-\frac{Y}{b}-\varepsilon M_{0}\right)^{2}+4 \varepsilon M_{0}}\right) \equiv M_{\max } \\
S^{\prime}(\xi)>0, \quad A^{\prime}(\xi)<0
\end{gathered}
$$

Proof. In view of (2.10)-(2.11), both $S$ and $A$ satisfy the classical elliptic strong maximum principle; see, for example, [9]. The maximum and minimum of $S$ and $A$ are achieved at the end points. Hence, (2.14) follows from (2.7). To show that $M \geq M_{0} \forall \xi \in[-d, d]$, suppose that $M<M_{0}$ at some point $\xi_{0} \in(-d, d)$ for some $\tau=\tau_{1} \in(0,1)$. Then, since $M$ is continuous in $\tau$ and $\xi \in[-d, d]$, as $\tau$ varies, the minimum of $M$ must pass through the interval $\left(0, M_{0}\right)$ before $M$ becomes negative. Let us assume that at $\tau_{1}, \min _{\xi \in[-d, d]} M \in\left(0, M_{0}\right)$. Hence, $\exists \xi_{1} \in(-d, d)$ and $0<$ 
$M\left(\xi_{1}\right)=\min _{\xi \in[-d, d]} M(\xi)<M_{0}$, for $\tau=\tau_{1}$. Evaluating $(2.12)$ at $\xi=\xi_{1}$ and $\tau=\tau_{1}$, we have

$$
-Y \tau_{1} R_{s, \varepsilon}^{(3)}\left(\xi_{1}\right)+b\left(M\left(\xi_{1}\right)-M_{0}\right)=\varepsilon M_{\xi \xi}\left(\xi_{1}\right)+c M_{\xi}\left(\xi_{1}\right) .
$$

At the minimum, the first derivative is zero and the second derivative is nonnegative, which results in

$$
-Y \tau_{1} R_{s, \varepsilon}^{(3)}\left(\xi_{1}\right)=\varepsilon M_{\xi \xi}\left(\xi_{1}\right)+b\left(M_{0}-M\left(\xi_{1}\right)\right)>0 .
$$

Since $Y, \tau_{1}>0$, we have that $R_{s, \varepsilon}^{(3)}\left(\xi_{1}\right)<0$. This is impossible since $M\left(\xi_{1}\right)>0$. It follows that $M \geq M_{0} \forall \tau \in[0,1], \xi \in[-d, d]$. By now, since $A \geq 0, S \geq 0, M \geq M_{0}$, we can identify $R_{s, \varepsilon}^{(1)}=R_{s, \varepsilon}^{(2)}=R_{s, \varepsilon}^{(3)} \equiv R_{s, \varepsilon}$.

To prove the upper bound in (2.15), we define $\max _{\xi \in[-d, d]} M(\xi)=M^{*} \geq M_{0}$. We only need to consider the case $M^{*}>M_{0}$. There exists $\xi^{*} \in(-d, d)$ such that $M\left(\xi^{*}\right)=M^{*}, M^{\prime}\left(\xi^{*}\right)=0, M^{\prime \prime}\left(\xi^{*}\right) \leq 0$. Evaluating (2.12) at $\xi=\xi^{*}$ implies that

$$
b\left(M^{*}-M_{0}\right) \leq\left.\frac{Y \tau A S M^{*}}{(1+A)\left(1+\varepsilon M^{*}\right)(1+S)}\right|_{\xi=\xi^{*}} \leq \frac{Y M^{*}}{1+\varepsilon M^{*}} .
$$

This can be rewritten as $b\left(M^{*}-M_{0}\right)\left(1+\varepsilon M^{*}\right) \leq Y M^{*}$, or

$$
M^{*} \leq \frac{1}{2 \varepsilon}\left(\varepsilon M_{0}+\frac{Y}{b}-1+\sqrt{\left(\varepsilon M_{0}+\frac{Y}{b}-1\right)^{2}+4 \varepsilon M_{0}}\right)=M_{\max }
$$

Notice that if $\frac{Y}{b} \geq 1$, then the right-hand side of (2.18) behaves like $O\left(\varepsilon^{-1}\right)\left(O\left(\varepsilon^{-1 / 2}\right)\right.$ if $Y=b)$ as $\varepsilon \rightarrow 0$. If $\frac{Y}{b}<1$, then in the limit $\varepsilon \rightarrow 0$, the right-hand side converges to $\frac{M_{0}}{1-b^{-1} Y}>M_{0}$. To prove (2.16), we rewrite (2.4) by multiplying both sides of the equation by $e^{\frac{\left(c R_{f}-v\right) \xi}{D}}$. Then, integrating from $-d$ to $\xi$, we obtain

$$
e^{\frac{\left(c R_{f}-v\right) \xi}{D}} S^{\prime}(\xi)=e^{\frac{-\left(c R_{f}-v\right) d}{D}} S^{\prime}(-d)+\int_{-d}^{\xi} \frac{1}{D} e^{\frac{\left(c R_{f}-v\right) \xi^{\prime}}{D}} R_{s}\left(\xi^{\prime}\right) d \xi^{\prime} .
$$

Since $S$ is not identically constant and, as a result of the Hopf lemma, it is clear that $S^{\prime}(-d)>0$. Thus, the entire right-hand side is positive and $S^{\prime}>0$. Similarly, it can be shown that $A^{\prime}<0$. The proof of the proposition is complete.

Proposition 2.2. Let $\tau \in[0,1]$ and let $d>1$ be fixed. There exists a constant $\underline{c}$ independent of $\tau \in[0,1]$ such that the wave speed $c=c(\tau)$ in $(2.10)-(2.12)$ satisfies

$$
\underline{c} \leq c(\tau) \leq v+\frac{D}{d} \ln \left(\frac{1}{\theta_{0}}-1\right)
$$

where $\theta_{0}=\frac{\theta}{A_{-}} \in(0,1)$.

Proof. To establish the upper bound for $c$, we find an upper solution $\bar{A}(\xi)$ to $A(\xi)$ and use it to bound $c$ from above. The upper solution solves

$$
\left\{\begin{array}{l}
D \bar{A}^{\prime \prime}+(c-v) \bar{A}^{\prime}=0, \quad \\
\bar{A}(-d)=A_{-}, \quad \bar{A}(d)=0
\end{array} \quad \xi \in[-d, d]\right.
$$


and is given by

$$
\bar{A}(\xi)=\frac{A_{-}\left(1-e^{-\left(\frac{c-v}{D}\right)(\xi-d)}\right)}{1-e^{\left(\frac{c-v}{D}\right) 2 d}} .
$$

By definition, $A(\xi) \leq \bar{A}(\xi)$, and in particular $A(0) \leq \bar{A}(0)$. Evaluating $\bar{A}$ at $\xi=0$ and solving for $c$, we have

$$
c \leq v+\frac{D}{d} \ln \left(\frac{A_{-}}{\theta}-1\right)=v+\frac{D}{d} \ln \left(\frac{1}{\theta_{0}}-1\right) .
$$

With (2.22), we have established the upper bound for the wave speed $c(\tau)$. To show the lower bound, we proceed similarly by defining a lower solution $\underline{A}(\xi)$ for $A(\xi)$ on $[-d, d]$ which solves

$$
D \underline{A}^{\prime \prime}+(c-v) \underline{A}^{\prime}=\left(\left[S^{+}-S(0, d)\right] H(\xi)+S(0, d)\right) F \underline{A},
$$

where

$$
F=F(\varepsilon) \equiv \frac{M_{\max }}{1+\varepsilon M_{0}} \geq \frac{M}{1+\varepsilon M} ; \quad H(\xi)= \begin{cases}1, & \xi \geq 0, \\ 0, & \xi<0,\end{cases}
$$

along with boundary and regularity conditions

$$
\underline{A}(-d)=A_{-}, \quad \underline{A}(d)=0, \quad \underline{A} \in C^{1} .
$$

It follows from (2.23) that

$$
\underline{A}(\xi)= \begin{cases}c_{1} e^{r_{1} \xi}+c_{2} e^{r_{2} \xi}, & \xi \in[-d, 0], \\ c_{3} e^{r_{3} \xi}+c_{4} e^{r_{4} \xi}, & \xi \in[0, d],\end{cases}
$$

where $r_{1,2}$ and $r_{3,4}$ are given by

$$
\begin{aligned}
& r_{1,2}=\frac{-(c-v) \pm \sqrt{(c-v)^{2}+4 D F S(0, \tau)}}{2 D}, \quad r_{1}>0, r_{2}<0 ; \\
& r_{3,4}=\frac{-(c-v) \pm \sqrt{(c-v)^{2}+4 D F S^{+}}}{2 D}, \quad r_{3}>0, r_{4}<0 .
\end{aligned}
$$

Using boundary conditions $(2.24)$ and the fact that $\underline{A}\left(0^{+}\right)=\underline{A}\left(0^{-}\right)$and $\underline{A}^{\prime}\left(0^{-}\right)=$ $\underline{A}^{\prime}\left(0^{+}\right)$, we solve for the constants

$$
\begin{aligned}
c_{1} & =A_{-} e^{r_{1} d}-c_{2} e^{\left(r_{1}-r_{2}\right) d}, \\
(2.28) c_{2} & =\frac{A_{-} e^{r_{1} d}\left[\left(r_{4}-r_{3} e^{\left(r_{4}-r_{3}\right) d}\right)-r_{1}\left(1-e^{\left(r_{4}-r_{3}\right) d}\right)\right]}{\left(r_{2}-r_{1} e^{\left(r_{1}-r_{2}\right) d}\right)\left(1-e^{\left(r_{4}-r_{3}\right) d}\right)-\left(r_{4}-r_{3} e^{\left(r_{4}-r_{3}\right) d}\right)\left(1-e^{\left(r_{1}-r_{2}\right) d}\right)}, \\
c_{3} & =-c_{4} e^{\left(r_{4}-r_{3}\right) d} \\
c_{4} & =\frac{A_{-} e^{r_{1} d}+c_{2}\left(1-e^{\left(r_{1}-r_{2}\right) d}\right)}{1-e^{\left(r_{4}-r_{3}\right) d}} .
\end{aligned}
$$

The solution is

$(2.29) \underline{A}(\xi)= \begin{cases}A_{-} e^{r_{1}(\xi+d)}+c_{2}\left(e^{r_{2} \xi}-e^{r_{1} \xi} e^{\left(r_{1}-r_{2}\right) d}\right), & \xi \in[-d, 0], \\ \left(\frac{A_{-} e^{r_{1} d}+c_{2}\left(1-e^{\left(r_{1}-r_{2}\right) d}\right)}{1-e^{\left(r_{4}-r_{3}\right) d}}\right)\left(e^{r_{4} \xi}-e^{\left(r_{4}-r_{3}\right) d} e^{r_{3} \xi}\right), & \xi \in[0, d],\end{cases}$ 
where $c_{2}$ is given in (2.28).

To find the lower bound for $c$, we assume there is no lower bound $(c \rightarrow-\infty)$ and use the following two properties to deduce a contradiction: $\underline{A}(0) \leq \theta, \underline{A}(0)=$ $A_{-} e^{r_{1} d}+c_{2}\left(1-e^{\left(r_{1}-r_{2}\right) d}\right)$. Combining these with equations $(2.28)-(2.29)$, we find that

$\theta \geq \underline{A}(0, \tau)$

$$
=\frac{A_{-} e^{r_{2} d}\left(1-e^{\left(r_{4}-r_{3}\right) d}\right)\left(r_{2}-r_{1}\right)}{\left(r_{2} e^{\left(r_{2}-r_{1}\right) d}-r_{1}\right)\left(1-e^{\left(r_{4}-r_{3}\right) d}\right)-\left(r_{4}-r_{3} e^{\left(r_{4}-r_{3}\right) d}\right)\left(e^{\left(r_{2}-r_{1}\right) d}-1\right)} .
$$

Fix any $\tau \in[0,1]$, and let $c \rightarrow-\infty$. We have from (2.26)-(2.27) that $r_{1} \rightarrow+\infty, r_{2} \rightarrow$ $0^{-}, r_{3} \rightarrow+\infty$, and $r_{4} \rightarrow 0^{-}$. It follows from $(2.30)$ that $\lim _{c \rightarrow-\infty} \underline{A}(0, \tau)=A_{-} \leq \theta$, contradicting the fact that $\theta \in\left(0, A_{-}\right)$. Since $S(0, \tau)$ is bounded between zero and $S_{+}$, it is easy to see that the limit of $A(0, \tau)$ as $c \rightarrow-\infty$ is uniform in $\tau \in[0,1]$. Hence, there exists a constant $\underline{c}$ independent of $\tau \in[0,1]$ such that $c(\tau) \geq \underline{c}$. The proof is complete.

Remark 2.1. By Propositions 2.1 and 2.2 and by standard elliptic estimates, the maximum norms of the derivatives of solutions $\left(S^{\prime}, A^{\prime}, M^{\prime}\right)$ are bounded independently of the parameter $\tau \in[0,1]$.

Next we show the existence of solutions to (2.10)-(2.12), (2.7), and (2.8) on the bounded domain $[-d, d]$ by Leray-Schauder degree theory. The idea is to transform the system of equations into a fixed point problem. The solution of the fixed point problem solves the original system of equations $(\tau=1)$ which is topologically equivalent to a simpler system $(\tau=0)$ for which we can easily determine the degree. Thus, showing that the degree is not zero amounts to proving the existence of a solution to the original system $(\tau=1)$. The proof is similar to that given in [1]; thus, the definitions and propositions are listed but the details of the proof can be found by referring to [1].

We begin by fixing $d$ and defining $I_{d}=[-d, d]$ and $X=\left(C^{1}\left(I_{d}\right)\right)^{3} \times R$. The set $X$ is a Banach space equipped with the norm

$$
\|(S, A, M, c)\|_{X} \equiv \max \left(\|S\|_{C^{1}\left(I_{d}\right)},\|A\|_{C^{1}\left(I_{d}\right)},\|M\|_{C^{1}\left(I_{d}\right)},|c|\right) .
$$

For each element $(S, A, M, c) \in X$, we consider the unique solution $(\bar{S}, \bar{A}, \bar{M})$ of the following system indexed by $\tau \in[0,1]$ :

$$
\begin{array}{r}
D \bar{S}_{\xi \xi}+\left(R_{f} c-v\right) \bar{S}_{\xi}=\tau \frac{q S A M}{(1+S)(1+A)(1+\varepsilon M)}, \\
D \bar{A}_{\xi \xi}+(c-v) \bar{A}_{\xi}=\gamma \tau \frac{q S A M}{(1+S)(1+A)(1+\varepsilon M)}, \\
\varepsilon \bar{M}_{\xi \xi}+c \bar{M}_{\xi}-b\left(\bar{M}-M_{0}\right)=-Y \tau \frac{q S A M}{(1+S)(1+A)(1+\varepsilon M)}
\end{array}
$$

subject to the boundary and normalization conditions (2.7) and (2.8). We define a compact map $K_{\tau}:(S, A, M, c) \in X \rightarrow(\bar{S}, \bar{A}, \bar{M}, c-\bar{A}(0)+\theta) \in X$. Every solution of (2.10)-(2.12) is a fixed point of the map $K_{\tau}$ given by $K_{\tau}[(S, A, M, c)]=$ $(S, A, M, c)$. We define another map, $F_{\tau}=I-K_{\tau}$, where $I$ is the identity map in $X$ $F_{\tau}:(S, A, M, c) \rightarrow(S-\bar{S}, A-\bar{A}, M-\bar{M}, \bar{A}(0)-\theta)$. Every solution to (2.10)-(2.12) is a solution of $F_{\tau}=0$.

The set $\Omega$, upon which the degree is well defined, is

$$
\Omega=\left\{(S, A, M, c) \in X\left|\|S\|_{C^{1}} \leq N,\|A\|_{C^{1}} \leq N,\|M\|_{C^{1}} \leq N,\right| c \mid \leq N\right\}
$$


where $N$ is larger than the $\tau$-independent a priori bounds of $C^{1}$ norms of $(S, A, M)$ in Proposition 2.1 and Remark 2.1; $N$ is also larger than the absolute value of the upper and lower bounds of $c(\tau)$ given in Proposition 2.2. The degree of $F_{\tau}$ at 0 , or $\operatorname{deg}\left(F_{\tau}, \bar{\Omega}, 0\right)$, is known to be well defined if $F_{\tau}(\partial \Omega) \neq 0$.

Proposition 2.3. For all $\tau \in[0,1], F_{\tau}(\partial \Omega)=\left(I-K_{\tau}\right)(\partial \Omega) \neq 0$.

Proof. See Proposition 7.3 in [1].

Proposition 2.4. $F_{\tau}=\left(I-K_{\tau}\right)$ satisfies, $\forall \tau \in[0,1]$,

$$
\operatorname{deg}\left(F_{\tau}, \bar{\Omega}, 0\right)=\operatorname{deg}\left(F_{0}, \bar{\Omega}, 0\right)=-1 .
$$

Proof. Equations (2.13) are the solutions denoted in this proof by $\left(S^{0}, A^{0}, M^{0}\right)$. See Proposition 7.5 in [1] for more details.

3. Further estimates on the regularized system. In this section, we obtain further estimates on solutions of the system (2.4)-(2.8) which enable us to extend the solutions to the entire real line in section 4 . In particular, by carefully choosing the value of $\theta$, we find the correct wave speed and prevent solutions from converging to the trivial solution as $d \rightarrow \infty$.

To begin, we rewrite the system of equations as

$$
\begin{array}{r}
D S_{\xi \xi}+\left(R_{f} c-v\right) S_{\xi}=R_{s, \varepsilon}, \\
D A_{\xi \xi}+(c-v) A_{\xi}=\gamma R_{s, \varepsilon}, \\
\varepsilon M_{\xi \xi}+c M_{\xi}=-Y R_{s, \varepsilon}+b\left(M-M_{0}\right),
\end{array}
$$

where $\varepsilon \in(0,1)$. The boundary conditions are

$$
S(-d)=A(d)=0, S(d)=S^{+}, A(-d)=A_{-}, M( \pm d)=M_{0},
$$

and the normalization condition is

$$
A(0)=\theta, \quad \theta \in\left(0, A_{-}\right) .
$$

The modified reaction term, $R_{s, \varepsilon}$, is

$$
R_{s, \varepsilon}=\frac{M S A}{(1+\varepsilon M)(1+S)(1+A)} .
$$

Proposition 3.1. There exist $\theta_{0}^{*} \in(0,1)$ and constants $\alpha, d_{0}>0$ independent of $d$, such that if $\theta_{0} \in\left(0, \theta_{0}^{*}\right), \theta=\theta_{0} A_{-}$, and $d \geq d_{0}$, the wave speed $c$ in (3.1)-(3.3) satisfies

$$
\frac{D}{d R_{f}} \ln \alpha+\frac{v}{R_{f}}-\frac{D}{d R_{f}}\left[\ln d+\ln S^{+}\right] \leq c \leq v+\frac{D}{d} \ln \left(\frac{1}{\theta_{0}}-1\right),
$$

implying in the limit $d \rightarrow \infty$

$$
\frac{v}{R_{f}} \leq \liminf _{d \rightarrow \infty} c \leq \limsup _{d \rightarrow \infty} c \leq v
$$

Proof. In view of Proposition 2.2, we need only to establish the lower bound of (3.6). To find the lower bound, we use the same approach as in Proposition 2.2 but consider an upper solution for $S$ (rather than $A$ ) which satisfies

$$
\left\{\begin{array}{l}
D \bar{S}^{\prime \prime}+\left(c R_{f}-v\right) \bar{S}^{\prime}=0, \quad \xi \in[-d, d] \\
\bar{S}(-d)=0, \quad \bar{S}(d)=S^{+}
\end{array}\right.
$$


Solving this differential equation and evaluating it at $\xi=0$, we obtain the inequality

$$
c \geq \frac{v}{R_{f}}-\frac{D}{R_{f} d} \ln \left(\frac{S^{+}}{S(0)}-1\right),
$$

where $S(0)=S(0, d)$, due to the implicit dependence of $S(0)$ on $d$. To find the lower bound for $c$, we must bound $S(0, d)$ from below. Defining $L=\liminf _{d \rightarrow \infty} S(0, d) d$, consider the following two cases for $L$. First, suppose that $0<L \leq \infty$. Then, $S(0, d) \geq \frac{\alpha}{d}$ for $\alpha \in\left(0, \frac{L}{2}\right)$, and $d$ large enough. By (3.9),

$$
c \geq \frac{v}{R_{f}}-\frac{D}{R_{f} d} \ln \left[\frac{d S^{+}}{\alpha}-1\right] \geq \frac{v}{R_{f}}-\frac{D}{d R_{f}}\left[\ln d+\ln S^{+}\right]+\frac{D}{d R_{f}} \ln \alpha,
$$

and so, for $0<L \leq \infty$, the proposition is proved.

Now suppose that $L=0$; then $\forall \delta>0, \exists d(\delta)$ such that if $d \geq d(\delta), S(0, d) \leq$ $\frac{\alpha}{d}$. Since $\lim \sup _{d \rightarrow \infty} c \leq v$, we can assume that $\liminf _{d \rightarrow \infty} c \leq \frac{v}{R_{f}}$; otherwise the proposition holds for large enough $d$. Therefore, there exists a sequence $\left\{d_{j}\right\} \rightarrow \infty$, such that $c\left(d_{j}\right) \rightarrow c^{*}$, with $c^{*} \leq \frac{v}{R_{f}}$.

Evaluating the lower solution $\underline{A}(\xi)$ in the proof of Proposition 2.2 (with $\tau=1$, $\left.d=d_{j}\right)$ at $\xi=0$, we have $\underline{A}(0)=A_{-} e^{r_{1} d_{j}}+c_{2}\left(1-e^{\left(r_{1}-r_{2}\right) d_{j}}\right)$, where $r_{1,2}$ and $r_{3,4}$ are given by $(2.26)-(2.27)$ with $S(0, \tau)$ replaced by $S\left(0, d_{j}\right)$, and $c_{2}$ as in $(2.28)$. It follows that

$$
\begin{aligned}
\lim _{j \rightarrow \infty} r_{1} & =\frac{v-c^{*}}{D}, \lim _{j \rightarrow \infty} r_{2}=0, \\
\lim _{j \rightarrow \infty} r_{3,4} & =\frac{\left(v-c^{*}\right) \pm \sqrt{\left(v-c^{*}\right)^{2}+4 D F S^{+}}}{2 D} .
\end{aligned}
$$

Using the assumption that $L=0$,

$$
\lim _{j \rightarrow \infty} r_{2} d_{j}=\lim _{j \rightarrow \infty} \frac{-2 F S\left(0, d_{j}\right) d_{j}}{(v-c)+\sqrt{(v-c)^{2}+4 D F S\left(0, d_{j}\right)}}=0 .
$$

Therefore, we infer from (3.11)-(3.12) that

$$
\theta \geq \lim _{j \rightarrow \infty} \underline{A}(0) \geq A_{-} \frac{2}{1+\sqrt{1+\frac{4 D F S+}{v^{2}\left(1-\frac{1}{R_{f}}\right)^{2}}}} .
$$

Recall that $\theta=\theta_{0} A_{-}$. We see from (3.13) that

$$
\theta_{0} \geq \theta_{0}^{*} \equiv \frac{2}{1+\sqrt{1+\frac{4 D F S^{+}}{v^{2}\left(1-R_{f}^{-1}\right)^{2}}}} .
$$

Since $\theta_{0} \in(0,1)$, we can choose $\theta_{0}<\theta_{0}^{*}$ to deduce a contradiction. So for large $d$ and $\theta_{0}<\theta_{0}^{*}$, we conclude that $L>0$. We have proven Proposition 3.1.

COROLlary 3.1. If $\liminf _{d \rightarrow \infty} c=\frac{v}{R_{f}}$ and $\theta \in\left(0, \theta_{0}^{*}\right)$, then

$$
\liminf _{d \rightarrow \infty} S(0, d) d>0 .
$$


Proof. In view of (3.12), the proof above implies that $\liminf _{d \rightarrow \infty} S(0, d) d>0$ which is equivalent to saying that there exists a constant $\alpha=\alpha(\varepsilon)>0$, such that for $d$ large, $S(0, d) \geq \alpha d^{-1}$. In other words, if $S(0, d)$ tends to zero, then it goes to zero more slowly than $\frac{1}{d}$.

Lemma 3.1. If $\liminf _{d \rightarrow \infty} c=\frac{v}{R_{f}}$ and $\theta \in\left(0, \theta_{0}^{*}\right)$, then the derivative $A^{\prime}(d)$ satisfies

$$
\begin{aligned}
\limsup _{d \rightarrow \infty}\left|A^{\prime}(d)\right| & \leq \frac{\theta}{D} \limsup _{d \rightarrow \infty} \sqrt{(v-c)^{2}+\frac{4 \gamma D S(0, d) M_{0}}{\left(1+S^{+}\right)\left(1+A_{-}\right)}} \\
& \leq \frac{\theta}{D} \sqrt{\left(v-\frac{v}{R_{f}}\right)^{2}+\frac{4 \gamma D M_{0}}{1+A_{-}}}
\end{aligned}
$$

Proof. First, notice that $\forall \xi \in[0, d]$, we have $\gamma R_{s} \geq \eta_{1}(d) A$, where

$$
\eta_{1}(d)=\frac{\gamma S(0, d) M_{0}}{\left(1+S^{+}\right)\left(1+A_{-}\right)\left(1+\varepsilon M_{\max }\right)} \geq O\left(d^{-1}\right)
$$

and the lower bound is given by Corollary 3.1. Now, define $\bar{A}(\xi)$ to solve

$$
\left\{\begin{array}{l}
D \bar{A}^{\prime \prime}+(c-v) \bar{A}^{\prime}-\eta_{1}(d) \bar{A}=0, \quad \xi \in[0, d], \\
\bar{A}(0)=\theta, \quad \bar{A}(d)=0 .
\end{array}\right.
$$

Solving (3.15),

$$
\bar{A}(\xi)=\theta e^{r_{2} \xi} \frac{e^{\left(r_{1}-r_{2}\right) d}-e^{\left(r_{1}-r_{2}\right) \xi}}{e^{\left(r_{1}-r_{2}\right) d}-1}, r_{1,2}=\frac{v-c \pm \sqrt{(v-c)^{2}+4 \eta_{1}(d) D}}{2 D} .
$$

By the assumptions, Corollary 3.1, and (3.14), we see that $-\left(r_{2}-r_{1}\right) d \rightarrow+\infty$, so $e^{\left(r_{2}-r_{1}\right) d} \rightarrow 0$ and $e^{r_{2} d} \leq 1$. Therefore, by Proposition 3.1,

$$
\lim _{d \rightarrow \infty}\left|A^{\prime}(d)\right| \leq \lim _{d \rightarrow \infty}\left|\bar{A}^{\prime}(d)\right| \leq \frac{\theta}{D} \sqrt{\left(v-\frac{v}{R_{f}}\right)^{2}+\frac{4 D M_{0} \gamma}{1+A_{-}}} .
$$

The proof is complete.

Note that if $\liminf _{d \rightarrow \infty} \eta_{1}(d)>0, \lim _{d \rightarrow \infty} e^{r_{2} d}=0$. Then, $\lim _{d \rightarrow \infty} A^{\prime}(d)=0$. However, we need more results to deduce that $\eta_{1}(d)$, or rather $S(0, d)$, does not converge to zero as $d \rightarrow \infty$.

Proposition 3.2. If $\theta \in\left(0, \theta_{0}^{*}\right)$, then $\lim _{d \rightarrow \infty} S^{\prime}(-d)=\lim _{d \rightarrow \infty} A^{\prime}(-d)=0$.

Proof. Step 1. $\lim _{d \rightarrow \infty}\left|S^{\prime}(-d)\right|=0$. To prove this, we find an upper solution to $S$ and use a resulting inequality to bound $\lim _{d \rightarrow \infty}\left|S^{\prime}(-d)\right|$ by zero on both sides. To construct an upper solution, we note that on $[-d, 0], R_{s} \geq \eta_{2} S$, where we define $\eta_{2}=\frac{M_{0} \theta}{\left(1+\varepsilon M_{\max }\right)\left(1+A_{-}\right)\left(1+S^{+}\right)}$, which is a constant, independent of $d$. Let $\bar{S}$ solve (3.8) with the right-hand side equal to $\eta_{2} \bar{S}$ and $\bar{S}(-d)=0, \bar{S}(0)=S(0, d)$ for $\xi \in[-d, 0]$. Solving for $\bar{S}$, we obtain

$$
\bar{S}(\xi)=S(0, d) e^{\bar{r}_{1} \xi}\left(\frac{e^{\left(\bar{r}_{1}-\bar{r}_{2}\right) d}-e^{\left(\bar{r}_{2}-\bar{r}_{1}\right) \xi}}{e^{\left(\bar{r}_{1}-\bar{r}_{2}\right) d}-1}\right), \quad \xi \in[-d, 0],
$$

where

$$
\bar{r}_{1,2}=\frac{\left(v-c R_{f}\right)}{2 D} \pm \frac{\sqrt{\left(v-c R_{f}\right)^{2}+4 D \eta_{2}}}{2 D}, \quad \bar{r}_{1}>0, \bar{r}_{2}<0
$$




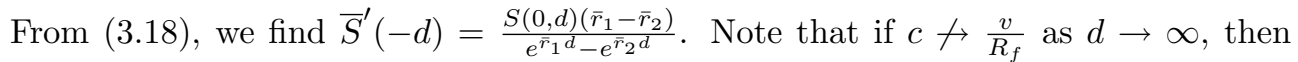
by Proposition 3.1, $c>\frac{v}{R_{f}}$ for large $d$, and so $\bar{r}_{2}<-\sqrt{\eta_{2} / D}$ for large $d$. If $c \rightarrow \frac{v}{R_{f}}$, as $d \rightarrow \infty$, then $\bar{r}_{2} \rightarrow-\sqrt{\eta_{2} / D}$. In either case, $e^{\bar{r}_{2} d}$ converges to zero, and $e^{\bar{r}_{1} d}$ converges to infinity. Thus, $0 \leq \lim \sup _{d \rightarrow \infty} S^{\prime}(-d) \leq \lim \sup _{d \rightarrow \infty} \bar{S}^{\prime}(-d)=0$, and the proof of Step 1 is complete.

Step 2. $\lim _{d \rightarrow \infty} A^{\prime}(-d)=0$. We infer from (3.18) that

$$
\bar{S}(\xi) \leq S(0, d) e^{\bar{r}_{1} \xi} \frac{e^{\left(\bar{r}_{1}-\bar{r}_{2}\right) d}}{e^{\left(\bar{r}_{1}-\bar{r}_{2}\right) d}-1} \leq 2 S^{+} e^{\bar{r}_{1} \xi} \quad \forall \xi \in[-d, 0],
$$

where $\bar{r}_{1}$ is bounded away from zero uniformly in $d \rightarrow \infty$. Using (3.19), we find an upper bound for $R_{s}$ to be $\gamma R_{s} \leq \eta_{3}(d) A \forall \xi \in[-d, 0]$, where $\eta_{3}$ is given in (3.20). We define a lower solution, $\underline{A}$, as the solution of

$$
\begin{cases}D \underline{A}^{\prime \prime}+(c-v) \underline{A}^{\prime}-\eta_{3} \underline{A}=0, & \eta_{3}(d)=\frac{2 \gamma S^{+} M_{\max } e^{-\bar{r}_{1} \frac{d}{2}}}{\left(1+\varepsilon M_{0}\right)(1+\theta)} . \\ \underline{A}(-d)=A_{-}, \quad \underline{A}\left(-\frac{d}{2}\right)=\theta \quad \forall \xi \in\left[-d,-\frac{d}{2}\right] .\end{cases}
$$

We solve (3.20) to get $\underline{A}(\xi)=c_{1} e^{r_{1} \xi}+c_{2} e^{r_{2} \xi}$, with $r_{1,2}$ given by (2.26) with $F S(0, \tau)$ replaced by $\eta_{3}(d)$. Differentiating $\underline{A}(\xi)$, we find

$$
\underline{A}^{\prime}(-d)=r_{1} A_{-}+\frac{\left(r_{1}-r_{2}\right) A_{-}+\left(r_{2}-r_{1}\right) \theta e^{-\frac{r_{1} d}{2}}}{e^{\left(r_{2}-r_{1}\right) \frac{d}{2}}-1} .
$$

Suppose that $c \rightarrow v$ along a subsequence $\left\{d_{j}\right\} \rightarrow \infty$; then $r_{1} \rightarrow 0$ and $r_{2} \rightarrow 0$. If, in addition $\left(r_{2}-r_{1}\right) d_{j} \rightarrow 0$, then $e^{\frac{\left(r_{2}-r_{1}\right) d_{j}}{2}}-1 \sim\left(r_{2}-r_{1}\right) \frac{d_{j}}{2}+O\left(\left(r_{2}-r_{1}\right)^{2} d_{j}^{2}\right)$, and $\underline{A}^{\prime}\left(-d_{j}\right) \rightarrow 0$. If $\liminf _{j \rightarrow \infty}\left(r_{2}-r_{1}\right) d_{j}>0$, then the second term in (3.21) with $d=d_{j}$ goes to zero because its numerator converges to zero while its denominator does not. The first term clearly converges to zero. Hence, $\underline{A}^{\prime}\left(-d_{j}\right) \rightarrow 0$ as $j \rightarrow \infty$.

Suppose now that $c \rightarrow c^{*}<v$ along a subsequence $d_{j} \rightarrow \infty$; then $r_{1} \rightarrow \frac{\left|c^{*}-v\right|}{D}$ and $r_{2} \rightarrow 0$ and also, $\left|r_{2}-r_{1}\right| \rightarrow \beta>0$, for a finite number $\beta$. Passing to the $d_{j} \rightarrow \infty$ limit in (3.21), we arrive at $\lim _{j \rightarrow \infty} \underline{A}^{\prime}\left(-d_{j}\right)=0$. It follows that we always have $\lim _{d \rightarrow \infty} A^{\prime}(-d)=0$. The proof is complete.

Lemma 3.2. Let $\theta \in\left(0, \theta_{0}^{*}\right)$. There exists a positive constant $K_{1}$ depending only on $D, v, R_{f}, \gamma, M_{\max }$ such that $\lim \sup _{d \rightarrow \infty} S^{\prime}(d) \leq K_{1} S^{+} \max (\theta, \sqrt{\theta})$.

Proof. In this proof, we construct a lower solution to $S$ on half the finite domain to find an inequality for $S^{\prime}$. To find the upper bound, we bound all the terms as $d \rightarrow \infty$. First, note that $A(\xi) \leq \theta \forall \xi \in[0, d]$. Next, define the subsolution $\underline{S}$ as

$$
\left\{\begin{array}{l}
D \underline{S}^{\prime \prime}+\left(c R_{f}-v\right) \underline{S^{\prime}-\eta_{4}} \underline{S}=0, \quad \xi \in[0, d], \\
\underline{S}(0)=S(0, d), \quad \underline{S}(d)=S^{+},
\end{array}\right.
$$

where $\eta_{4}=\theta K$ and $K \equiv \gamma M_{\max }$ since $\gamma R_{s} \leq \gamma \theta M_{\max } S$. Note that $\eta_{4}$ is a positive constant independent of $d$. The solution of (3.22) is $\underline{S}=c_{1} e^{r_{1} \xi}+c_{2} e^{r_{2} \xi}$, where $r_{1,2}=$ $\bar{r}_{1,2}$ from Proposition 2.2 with $\eta_{2}$ replaced by $\eta_{4}$. Solving (3.22) and differentiating, it follows that

$$
\underline{S}^{\prime}(d)=\frac{\left(r_{1}-r_{2}\right) S(0, d) e^{r_{2} d}-r_{1} S^{+}+r_{2} e^{\left(r_{2}-r_{1}\right) d} S^{+}}{e^{\left(r_{2}-r_{1}\right) d}-1},
$$

where $r_{2}$ is rewritten as

$$
r_{2}=\frac{-4 D \eta_{4}}{2 D \cdot\left(v-c R_{f}+\sqrt{\left(v-c R_{f}\right)^{2}+4 D \eta_{4}}\right)},
$$


and $r_{2}-r_{1}=-\frac{1}{D} \sqrt{\left(v-c R_{f}\right)^{2}+4 D \eta_{4}} \leq-\frac{1}{D} 2 \sqrt{D \theta K}$. Thus, $e^{\left(r_{2}-r_{1}\right) d} \rightarrow 0$ as $d \rightarrow \infty$. To find an upper bound on $S^{\prime}(d)$, we must bound $r_{1}$ and $r_{2}$. If $v-c R_{f} \leq 0$, then $\left|r_{2}\right| \geq \sqrt{\frac{\eta_{4}}{D}}=\sqrt{\frac{\theta K}{D}}$. If $v-c R_{f}>0$, by Proposition 3.1, we see that $v-c R_{f} \leq$ $O\left(d^{-1} \ln d\right)$. Hence, we find a lower bound for $r_{2}$ in the limit $d \rightarrow \infty$ as

$$
\left|r_{2}\right| \geq \frac{2 \eta_{4}}{O\left(d^{-1} \ln d\right)+\sqrt{\left(O\left(d^{-1} \ln d\right)\right)^{2}+4 D \eta_{4}}} \rightarrow \sqrt{\frac{\eta_{4}}{D}}
$$

and so $\lim \sup _{d \rightarrow \infty} r_{2} \leq-\sqrt{D^{-1} \eta_{4}}=-\sqrt{D^{-1} \theta K}$. Now, we see that $\lim _{d \rightarrow \infty} e^{r_{2} d} \leq$ $\lim _{d \rightarrow \infty} e^{-\sqrt{D^{-1} \theta K} d}=0$. We consider the same cases in order to bound $r_{1}$. If $v-$ $c R_{f} \leq 0$, then

$$
r_{1} \leq \frac{2 \eta_{4}}{\left(\left(v+O\left(\frac{1}{d}\right)\right) R_{f}-v\right)+\sqrt{\left(\left(v+O\left(\frac{1}{d}\right)\right) R_{f}-v\right)^{2}+4 D \eta_{4}}}
$$

by Proposition 3.1. Therefore,

$$
\limsup _{d \rightarrow \infty} r_{1} \leq \frac{2 \eta_{4}}{v\left(R_{f}-1\right)+\sqrt{v^{2}\left(R_{f}-1\right)^{2}+4 D \eta_{4}}} .
$$

If $v-c R_{f}>0$, then by Proposition 3.1 again $v-c R_{f} \leq O\left(d^{-1} \ln d\right)$ for large $d$, and so

$$
r_{1} \leq \frac{O\left(d^{-1} \ln d\right)+\sqrt{O\left(d^{-2}(\ln d)^{2}\right)+4 D \eta_{4}}}{2 D}
$$

and finally,

$$
\limsup _{d \rightarrow \infty} r_{1} \leq \sqrt{\frac{\theta K}{D}}
$$

In any case, we have the following:

$$
\limsup _{d \rightarrow \infty} r_{1} \leq 2 K_{1}\left(D, \gamma, v, R_{f}, M_{\max }\right) \max (\theta, \sqrt{\theta})=K_{1} \max (\theta, \sqrt{\theta}) .
$$

Combining (3.23) and (3.28), we obtain the result

$$
\limsup _{d \rightarrow \infty} S^{\prime}(d) \leq S^{+} \limsup _{d \rightarrow \infty} r_{1}=K_{1} S^{+} \max (\theta, \sqrt{\theta}),
$$

and the proof is complete.

LEMma 3.3. There exist two positive constants $\delta_{1}$ and $\delta_{2}$ independent of $d$, and a positive number $\theta_{0}^{* *}$ depending only on $\delta_{1}$ and $\delta_{2}, \theta_{0}^{* *} \in\left(0, \theta_{0}^{*}\right)$. If $\theta_{0} \in\left(0, \theta_{0}^{* *}\right)$, then

$$
\frac{v}{R_{f}}+\delta_{1} \leq \liminf _{d \rightarrow \infty} c \leq \limsup _{d \rightarrow \infty} c \leq v-\delta_{2} .
$$

Proof. Combining the equations for $S$ and $A$ in (3.1)-(3.2) and integrating from $-d$ to $d$, we obtain

$$
\gamma\left(D S^{\prime}(d)+\left(c R_{f}-v\right) S^{+}-D S^{\prime}(-d)\right)=D A^{\prime}(d)-D A^{\prime}(-d)-(c-v) A_{-} .
$$


We solve for $c$ to get

$$
c=\frac{D\left(A^{\prime}(d)-A^{\prime}(-d)\right)+\gamma D\left(S^{\prime}(-d)-S^{\prime}(d)\right)+v A_{-}+v \gamma S^{+}}{\gamma R_{f} S^{+}+A_{-}} .
$$

Using Proposition 2.1, we derive the inequality

$$
\frac{D A^{\prime}(d)-\gamma D S^{\prime}(d)+v A_{-}+v \gamma S^{+}}{\gamma R_{f} S^{+}+A_{-}} \leq c \leq \frac{-D A^{\prime}(-d)+\gamma D S^{\prime}(-d)+v A_{-}+v \gamma S^{+}}{\gamma R_{f} S^{+}+A_{-}} .
$$

Taking $d \rightarrow \infty$ and using Proposition 3.2, $\limsup _{d \rightarrow \infty} c<v$. If $\liminf _{d \rightarrow \infty} c>\frac{v}{R_{f}}$, then the proof is done if we define $\delta_{i}, i=1,2$, to be the difference between $c$ and $\frac{v}{R}$ and $v$, respectively. On the other hand, if $\liminf _{d \rightarrow \infty} c=\frac{v}{R_{f}}$, then by Lemmas 3.1 and 3.2 , we have

$$
\begin{aligned}
& \liminf _{d \rightarrow \infty} c \\
& \geq \frac{-\theta \sqrt{v^{2}\left(1-R_{f}^{-1}\right)^{2}+\frac{4 \gamma D M_{0}}{1+A_{-}}}-\gamma D K_{1} S^{+} \max \left(\theta, \theta^{\frac{1}{2}}\right)+v A_{-}+v \gamma S^{+}}{\gamma R_{f} S^{+}+A_{-}} .
\end{aligned}
$$

Now, set $\delta_{2}=\left(\gamma S^{+}\left(R_{f}-1\right) v\right) /\left(\gamma S^{+} R_{f}+A_{-}\right)>0$, and

$$
\delta_{1}=\frac{v\left(1-\frac{1}{R_{f}}\right)\left(A_{-}+S^{+} \gamma\right)-\theta \sqrt{v^{2}\left(1-R_{f}^{-1}\right)^{2}+\frac{4 \gamma D M_{0}}{1+A_{-}}}-\gamma D K_{1} S^{+} \max \left(\theta, \theta^{\frac{1}{2}}\right)}{\gamma S^{+} R_{f}+A_{-}} .
$$

Here $\delta_{1}$ is the difference between the lower bound in (3.32) and $\frac{v}{R_{f}}$. There exists a $\theta_{0}^{* *} \in\left(0, \theta_{0}^{*}\right)$ such that if $\theta_{0} \in\left(0, \theta_{0}^{* *}\right), \delta_{1}>0$. We end the proof.

LEMMA 3.4. There exists a positive constant, $\theta_{0}^{* * *}$, depending on $\delta_{1}, \delta_{2}$ and less than $\theta_{0}^{* *}$ such that if $\theta_{0} \leq \theta_{0}^{* * *}, \liminf _{d \rightarrow \infty} S(0, d)>0$.

Proof. Suppose $S\left(0, d_{j}\right) \rightarrow 0$ along a sequence $\left\{d_{j}\right\} \rightarrow \infty$; then $S(\xi) \rightarrow 0$, uniformly in $\xi \in\left[-d_{j}, 0\right]$. Proposition 3.1 says that $c_{j}=c\left(d_{j}\right)$ is uniformly bounded in $d_{j} ;(2.10)-(2.12)$ then imply that $(S, A, M)\left(\xi, d_{j}\right)$ is compact in $\left(C_{l o c}^{1}(R)\right)^{3}$. Up to a subsequence, we have $(S, A, M)\left(\xi, d_{j}\right) \rightarrow(\tilde{S}, \tilde{A}, \tilde{M})(\xi)$ as $j \rightarrow \infty, \tilde{S}^{\prime} \geq 0$, $\tilde{A}^{\prime} \leq$ $0, \tilde{A}(0)=\theta, \tilde{S}(\xi) \equiv 0$ if $\xi \leq 0$. By uniqueness of solutions to ordinary differential equations, we deduce from (2.10) that $\tilde{S} \equiv 0$ on $R^{1}$. Thus, $\tilde{A}$ is a bounded solution to the problem

$$
\left\{\begin{array}{l}
D \tilde{A}_{\xi \xi}+\left(c^{*}-v\right) \tilde{A}_{\xi}=0, \quad \xi \in R^{1} \\
\tilde{A}(0)=\theta
\end{array}\right.
$$

As a consequence of Lemma 3.3, along a subsequence of $d_{j} \rightarrow \infty, c\left(d_{j}\right) \rightarrow c^{*} \in$ $\left(\frac{v}{R_{f}}+\delta_{1}, v-\delta_{2}\right)$ as $j \rightarrow \infty$, where $\delta_{i}=\delta_{i}\left(D, v, R_{f}, \gamma, \theta_{0}, M_{0}, M_{\max }, S^{+}, A_{-}\right)>0$ for $i=1,2$. The only bounded solution of (3.33) is $\tilde{A} \equiv \theta$.

Let us consider the equations for $(S, A)$ on $\left[-d_{j}, d_{j}\right]$ :

$$
\begin{aligned}
& D S^{\prime \prime}+\left(c_{j} R_{f}-v\right) S^{\prime}=\frac{A}{1+A} \frac{M}{1+\varepsilon M} \frac{S}{1+S}, \\
& D A^{\prime \prime}+\left(c_{j}-v\right) A^{\prime}=\gamma \frac{A}{1+A} \frac{M}{1+\varepsilon M} \frac{S}{1+S} .
\end{aligned}
$$


Multiplying (3.34) by $\gamma$ and subtracting (3.35) from the resulting equation, we obtain

$$
\gamma D S^{\prime \prime}-D A^{\prime \prime}+\gamma\left(c_{j} R_{f}-v\right) S^{\prime}-\left(c_{j}-v\right) A^{\prime}=0, \quad \xi \in\left[-d_{j}, d_{j}\right] .
$$

Integrating once in $\xi$, we obtain

$$
\begin{aligned}
\gamma D S^{\prime}-D A^{\prime}+\gamma\left(c_{j} R_{f}-v\right) S-\left(c_{j}-v\right) A & =\gamma D S^{\prime}\left(d_{j}\right)-D A^{\prime}\left(d_{j}\right)+\gamma\left(c_{j} R_{f}-v\right) S^{+}, \\
& =Q\left(d_{j}\right)+\Gamma+o(1) \quad \forall \xi \in\left[-d_{j}, d_{j}\right],
\end{aligned}
$$

where $\Gamma=\lim _{j \rightarrow \infty} \gamma\left(c_{j} R_{f}-v\right) S^{+}$, and $Q\left(d_{j}\right)=\gamma D S^{\prime}\left(d_{j}\right)-D A^{\prime}\left(d_{j}\right) \geq 0$. It follows that

$$
\gamma D\left(S^{\prime}+\frac{c_{j} R_{f}-v}{D} S\right)=D A^{\prime}+\left(c_{j}-v\right) A+\Gamma+Q\left(d_{j}\right)+o(1),
$$

and so

$$
\gamma D\left(e^{\left(\frac{c_{j} R_{f}-v}{D}\right) \xi} S\right)^{\prime}=e^{\left(\frac{c_{j} R_{f}-v}{D}\right) \xi}\left[D A^{\prime}+\left(c_{j}-v\right) A+\Gamma+Q\left(d_{j}\right)+o(1)\right]
$$

Integrating (3.38) over $\left[-d_{j}, \xi\right]$ gives

$$
\begin{aligned}
& \gamma D e^{\left(\frac{c_{j} R_{f}-v}{D}\right) \xi} S=\int_{-d_{j}}^{\xi} e^{\left(\frac{c_{j} R_{f}-v}{D}\right) \xi^{\prime}}\left[D A^{\prime}+\left(c_{j}-v\right) A+\Gamma+Q\left(d_{j}\right)+o(1)\right] d \xi^{\prime} \\
& \geq D \int_{-d_{j}}^{\xi} e^{\left(\frac{c_{j} R_{f}-v}{D}\right) \xi^{\prime}} e^{\left(\frac{v-c_{j}}{D}\right) \xi^{\prime}} \cdot\left(e^{\left(\frac{c_{j}-v}{D}\right) \xi^{\prime}} A\right)^{\prime} d \xi^{\prime}+(\Gamma+o(1)) \int_{-d_{j}}^{\xi} e^{\left(\frac{c_{j} R_{f}-v}{D}\right) \xi^{\prime}} d \xi^{\prime} \\
& =D e^{\left(\frac{c_{j} R_{f}-v}{D}\right) \xi} A-D e^{-\left(\frac{c_{j} R_{f}-v}{D}\right) d_{j}} A_{-}-c_{j}\left(R_{f}-1\right) \int_{-d_{j}}^{\xi} e^{\left(\frac{c_{j} R_{f}-v}{D}\right) \xi^{\prime}} A\left(\xi^{\prime}\right) d \xi^{\prime} \\
& \quad+(\Gamma+o(1)) \frac{D}{c_{j} R_{f}-v} e^{\left(\frac{c_{j} R_{f}-v}{D}\right) \xi}+o(1) .
\end{aligned}
$$

Recall that if $S\left(0, d_{j}\right) \rightarrow 0$ as $d_{j} \rightarrow \infty$, then $A\left(\xi, d_{j}\right) \rightarrow \theta$ and $S\left(\xi, d_{j}\right) \rightarrow 0$ as $d_{j} \rightarrow \infty$, $\forall \xi$, uniformly for a compact set of $\xi$. It follows that by the dominated convergence theorem

$$
\begin{aligned}
\lim _{d_{j} \rightarrow \infty} \int_{-d_{j}}^{\xi} e^{\left(\frac{c_{j} R_{f}-v}{D}\right) \xi^{\prime}} A\left(\xi^{\prime}\right) d \xi^{\prime} & =\int_{-\infty}^{\xi} e^{\left(\frac{c^{*} R_{f}-v}{D}\right) \xi^{\prime}} \theta d \xi^{\prime} \\
& \left.=\theta \frac{D}{c^{*} R_{f}-v} e^{e^{c^{*} R_{f}-v}} \frac{\xi}{D}\right)
\end{aligned}
$$

so (3.39) in the limit $d_{j} \rightarrow \infty$ reads

$$
0 \geq D \theta_{0} A_{-}-c^{*} D \theta_{0} A_{-} \frac{\left(R_{f}-1\right)}{\left(c^{*} R_{f}-v\right)}+\frac{\Gamma D}{c^{*} R_{f}-v} .
$$

Substituting in $\Gamma=\gamma\left(c^{*} R_{f}-v\right) S^{+}$, we get $0 \geq \gamma S^{+} D+D \theta_{0} A_{-}\left[1-\frac{c^{*}\left(R_{f}-1\right)}{c^{*} R_{f}-v}\right]$, and finally

$$
0 \geq S^{+}+\gamma^{-1} \theta_{0} A_{-}\left(\frac{c^{*}-v}{c^{*} R_{f}-v}\right) \geq S^{+}-\gamma^{-1} \theta_{0} A_{-} \frac{\delta_{2}}{R_{f} \delta_{1}}
$$


There exists $\theta_{0}^{* * *} \in\left(0, \theta_{0}^{* *}\right)$ depending on $\delta_{1}$ and $\delta_{2}$ such that the right-hand side of (3.41) is strictly positive. This implies a contradiction. Therefore, $S(0, d)$ does not tend to zero as $d \rightarrow \infty$.

Proposition 3.3. If $\theta \in\left(0, \theta_{0}^{* * *}\right)$, then $\lim _{d \rightarrow \infty} A^{\prime}(d)=\lim _{d \rightarrow \infty} S^{\prime}(d)=0$.

Proof. Let us follow the proof of Lemma 3.1 until the derivative of $\bar{A}$ at $\xi=d$ is found to be

$$
\bar{A}^{\prime}(d)=\frac{\theta e^{r_{2} d}\left(r_{2}-r_{1}\right)}{1-e^{\left(r_{2}-r_{1}\right) d}},
$$

where $\left|r_{2}-r_{1}\right|$ is bounded. Now by Lemma 3.4, we can improve (3.14) so that $\liminf _{d \rightarrow \infty} \eta_{1}(d)>0$. Hence, $v-c$ is bounded strictly away from zero as $d \rightarrow \infty$ by Lemma 3.3, and so $\lim _{d \rightarrow \infty} e^{r_{2} d}=0$ and $\lim _{d \rightarrow \infty} A^{\prime}(d)=0$. $(3.16)$,

For the second part of the proposition, we consider $S(\xi, d)$ over $\left[\frac{d}{2}, d\right]$. First, by

$$
A(\xi) \leq \theta e^{\bar{r}_{2} \frac{d}{2}} \quad \forall \xi \in\left[\frac{d}{2}, d\right], \quad \bar{r}_{2}=\frac{v-c-\sqrt{(v-c)^{2}+4 \eta_{1}(d) D}}{2 D},
$$

and $\lim \sup _{d \rightarrow \infty} \bar{r}_{2}<0$. Hence, $A(\xi, d)$ goes to zero exponentially fast in $d$.

Let $\eta_{5}(d)=M_{\max } \theta e^{\bar{r}_{2} \frac{d}{2}}$, so that $R_{s} \leq \eta_{5} S$. Define a subsolution $\underline{S}$ on $\left[\frac{d}{2}, d\right]$ as in (3.22) with $\eta_{2}$ replaced by $\eta_{5}$ and $\underline{S}\left(\frac{d}{2}\right)=S(0, d), \underline{S}(d)=S^{+}$. Solving for $\underline{S}$, taking the derivative, and evaluating it at $\xi=d$, we get

$$
\underline{S}^{\prime}(d)=\frac{\left(r_{1}-r_{2}\right) S(0, d) e^{\frac{r_{2} d}{2}}-r_{1} S^{+}+r_{2} S^{+} e^{\left(r_{2}-r_{1}\right) \frac{d}{2}}}{e^{\left(r_{2}-r_{1}\right) \frac{d}{2}}-1} \geq S^{\prime}(d) \geq 0 .
$$

Since $c$ is bounded away from $\frac{v}{R_{f}}$ uniformly in $d$,

$$
\lim _{d \rightarrow \infty} e^{\left(r_{2}-r_{1}\right) \frac{d}{2}}=0, \lim _{d \rightarrow \infty} e^{r_{2} \frac{d}{2}}=0, \text { and } \lim _{d \rightarrow \infty} \eta_{5}(d)=0 .
$$

In addition, $\lim _{d \rightarrow \infty} r_{1}=0$, and we deduce immediately that $\lim _{d \rightarrow \infty} S^{\prime}(d)=0$. The proof is complete.

Proposition 3.4.

$$
\lim _{d \rightarrow \infty} c(d)=v \frac{\left(A_{-}+\gamma s^{+}\right)}{\left(A_{-}+\gamma R_{f} S^{+}\right)}=c .
$$

Proof. The limit follows from (3.31) in the proof of Lemma 3.3 and Propositions 3.2 and 3.3 .

COROLlary 3.2. There exist positive constants $\beta_{1}$ and $\beta_{2}$ independent of $d$ such that

$$
\begin{aligned}
& A(\xi, d) \leq \theta e^{-\beta_{1} \xi}, \quad \beta_{1}>0, \quad \forall \xi \in[0, d], \\
& S(\xi, d) \leq 2 S^{+} e^{\beta_{2} \xi}, \quad \beta_{2}>0, \quad \forall \xi \in[-d, 0] .
\end{aligned}
$$

Proof. To prove (3.45), we consider (3.16) from Lemma 3.1, defined on $[0, d]$. It is clear that $A(\xi, d) \leq \theta e^{r_{2} \xi}, \forall \xi \in[0, d]$. Note that $\lim _{\sup } \operatorname{sum}_{d \rightarrow \infty} r_{2}<0$. Letting $0<\beta_{1} \leq-\lim \sup _{d \rightarrow \infty} r_{2}$ with $\beta_{1}$ bounded away from zero uniformly in $d$, we have shown (3.45). To prove (3.46), we use (3.19): $S(\xi, d) \leq 2 S^{+} e^{\bar{r}_{1} \xi}, \forall \xi \in[-d, 0]$. We see that $\liminf \operatorname{in}_{d \rightarrow \infty} \bar{r}_{1}>0$. Choosing $0<\beta_{2} \leq \liminf _{d \rightarrow \infty} \bar{r}_{1}$, we have shown (3.46).

\section{$\square$}


4. Solutions of a regularized system on the real line. For a fixed $\varepsilon>0$, we are interested in taking the limit $d \rightarrow \infty$. The $\varepsilon$ dependence of solutions will not be specified until the next section. By Propositions 3.1-3.3, we know that the $d$ dependent solutions denoted by $\left(S_{d}, A_{d}, M_{d}\right)$ are compact in $\left(C_{l o c}^{1}(R)\right)^{3}$. So up to a subsequence in $d,\left(S_{d}, A_{d}, M_{d}\right) \rightarrow(S, A, M)$ uniformly on any compact set of $\xi$ and the limiting system is

$$
\begin{aligned}
& D S_{\xi \xi}+\left(R_{f} c-v\right) S_{\xi}=R_{s}, \\
& D A_{\xi \xi}+(c-v) A_{\xi}=\gamma R_{s}, \\
& \varepsilon M_{\xi \xi}+c M_{\xi}=-Y R_{s}+b\left(M-M_{0}\right),
\end{aligned}
$$

with boundary conditions

$$
A(0)=\theta_{0} A_{-}, \quad \theta_{0} \in\left(0, \theta_{0}^{* * *}\right), \quad S(0)=S(0, \varepsilon)>0 .
$$

Moreover, we have the following bounds:

$$
\begin{gathered}
0 \leq S(\xi) \leq S^{+}, \quad 0 \leq A(\xi) \leq A_{-}, \quad M_{0} \leq M(\xi) \leq M_{\max }, \\
A^{\prime}(\xi) \leq 0, \quad S^{\prime}(\xi) \geq 0 .
\end{gathered}
$$

Note that (4.4) is due to Lemma 3.4.

Corollary 3.2 holds for the limiting functions $A$ and $S$. In particular,

$$
\lim _{\xi \rightarrow+\infty} A(\xi)=0, \quad \lim _{\xi \rightarrow-\infty} S(\xi)=0 .
$$

Monotonicity of $A$ and $S$ in (4.6) implies that

$$
\begin{array}{ll}
\lim _{\xi \rightarrow-\infty} A(\xi)=\tilde{A}_{-} & \text {for } \tilde{A}_{-} \in\left(0, A_{-}\right], \\
\lim _{\xi \rightarrow+\infty} S(\xi)=\tilde{S}^{+} & \text {for } \tilde{S}^{+} \in\left(0, S^{+}\right] .
\end{array}
$$

Lemma 4.1. For $\varepsilon \in(0,1), \lim _{\xi \rightarrow \pm \infty} M(\xi)=M_{0}$.

Proof. Let $m=M-M_{0}$; then $m \geq 0$ and $m \neq \equiv 0$ (if $m \equiv 0$ by (4.3) $A S \equiv 0$ which contradicts $(4.4)$ at $\xi=0)$. Therefore, there exists a $\xi_{1}$ such that $\left(M-M_{0}\right)\left(\xi_{1}\right)=$ $m\left(\xi_{1}\right)>0$. On $\left[\xi_{1}, \infty\right)$ by inequalities (4.5) and Corollary 3.2 ,

$$
\frac{Y S A M}{(1+S)(1+A)(1+\varepsilon M)} \leq y_{0} Y M_{\max } \theta e^{-\beta_{1} \xi},
$$

where $y_{0}>1$ is to be chosen and

$$
\beta_{1} \leq \frac{c-v+\sqrt{(v-c)^{2}+4 \eta_{1}(d) D}}{2 D}, \quad \eta_{1}(d)>0 .
$$

Define $\bar{m}$ as a solution of $\varepsilon \bar{m}_{\xi \xi}+c \bar{m}_{\xi}-b \bar{m}=-y_{0} Y M_{\max } \theta e^{-\beta_{1} \xi}$ with $\bar{m}\left(\xi_{1}\right) \geq$ $m\left(\xi_{1}\right)>0$, for $\xi \in\left[\xi_{1}, \infty\right)$. Solving for a positive solution $\bar{m}$, we obtain

$$
\bar{m}=-y_{0} \frac{Y M_{\max } \theta}{\left(\varepsilon \beta_{1}^{2}-c \beta_{1}-b\right)} e^{-\beta_{1} \xi},
$$


where $\beta_{1}>0, \varepsilon \beta_{1}^{2}-c \beta_{1}-b<0$ for $\varepsilon \in(0,1)$ and $\beta_{1}<\sqrt{b}$. So indeed $\bar{m}>0$. We choose $y_{0}$ such that $\bar{m}\left(\xi_{1}\right) \geq m\left(\xi_{1}\right)$. By the maximum principle, $m(\xi) \leq \bar{m}(\xi)$. Since $\lim _{\xi \rightarrow+\infty} \bar{m}(\xi)=0$, we find that $\lim _{\xi \rightarrow+\infty} m(\xi)=0$ and $\lim _{\xi \rightarrow+\infty} M(\xi)=M_{0}$. A similar argument shows that $\lim _{\xi \rightarrow+\infty} M(\xi)=M_{0}$.

Lemma 4.2. Consider $M_{d}$ for $\xi \in[-d, d]$. Then $\lim _{d \rightarrow \infty} M_{d}^{\prime}( \pm d)=0$.

Proof. This proof is similar to the proof of Lemma 4.1. We define $m_{d}=M_{d}-M_{0}$ on $[-d, d]$ and then construct an upper solution to $m_{d}$ which solves

$$
\left\{\begin{array}{l}
\varepsilon \bar{m}_{d, \xi \xi}+c \bar{m}_{d, \xi}-b \bar{m}_{d}=-Y M_{\max } \theta e^{-\beta_{1} \xi}, \\
\bar{m}_{d}(0)=m_{d}(0), \quad \bar{m}_{d}(d)=0, \quad \xi \in[0, d],
\end{array}\right.
$$

where $c=c(d)$. We solve for $\bar{m}_{d}$ and find the derivative at $\xi=d$. Taking the limit as $d \rightarrow \infty$, we show that $\lim _{d \rightarrow+\infty} \overline{m^{\prime}} d(d)=0$. It follows that $\lim _{d \rightarrow+\infty}\left|m_{d}^{\prime}(d)\right|=$ $\lim _{d \rightarrow+\infty}\left|\left(M_{d}-M_{0}\right)^{\prime}(d)\right|=\lim _{d \rightarrow+\infty}\left|M_{d}^{\prime}(d)\right|=0$. A similar argument shows that $\lim _{d \rightarrow+\infty} M_{d}^{\prime}(-d)=0$.

LEMmA 4.3. There exists four constants $k_{1}, k_{2}, k_{3}$, and $k_{4}$ depending on $Y$, $M_{\max }, \theta, \varepsilon, b, c, R, v$, and $D$ such that

$$
\begin{aligned}
& \left(M-M_{0}\right)(\xi) \leq k_{1} e^{-\sigma_{1} \xi}+k_{2} e^{-\sigma_{2} \xi}, \quad \sigma_{1}>0, \sigma_{2}>0, \xi \geq 0, \\
& \left(M-M_{0}\right)(\xi) \leq k_{3} e^{\sigma_{3} \xi}+k_{4} e^{\sigma_{4} \xi}, \quad \sigma_{3}>0, \sigma_{4}>0, \xi \leq 0,
\end{aligned}
$$

where $\sigma_{i}$ is a constant for $i=1,2,3,4$.

Proof. The proof of (4.11) relies on bounding the upper solution $\bar{m}_{d}$ found in Lemma 4.2. The proof of (4.12) is similar.

TheOREM 4.1. For $\varepsilon \in(0,1), \exists$ a smooth solution $(S, A, M, c)$ solving the system (4.1)-(4.3) and the boundary conditions (4.4)-(4.6). Moreover, $M_{0}<M \leq M_{\max }$ and $\lim _{\xi \rightarrow \pm \infty} M(\xi)=M_{0}$.

Proof. We have shown that there exist solutions on the real line and that $M$ reaches its limits at the spatial infinities. Now, we only need to show that the boundary conditions on $S, A$ hold in the limit $d \rightarrow \infty$; i.e., we want to show that $A_{-}=\tilde{A}_{-}$ and $S^{+}=\tilde{S}^{+}$. To that end, we multiply $\gamma$ to (2.4), subtract (2.5), and integrate over $[-d, d]$. Taking the limit $d \rightarrow \infty$, using Propositions 3.2 and 3.3 and (4.7)-(4.8), we solve for $c$ :

$$
c=v \frac{\left(\tilde{A}_{-}+\gamma \tilde{S}^{+}\right)}{\left(\tilde{A}_{-}+\gamma \tilde{S}^{+} R_{f}\right)} .
$$

Proposition 3.4 says that $c=v \frac{\left(A_{-}+\gamma S^{+}\right)}{\left(A_{-}+\gamma S^{+} R_{f}\right)}$. Therefore, it is clear that $\frac{\tilde{S}^{+}}{S^{+}}=\frac{\tilde{A}_{-}}{A_{-}}$.

Multiplying (2.4) by $Y$, adding it to (2.6), and integrating over $[-d, d]$,

$Y D\left[S_{d}^{\prime}(d)-S_{d}^{\prime}(-d)\right]+Y\left(c_{d} R-v\right) S^{+}+\varepsilon\left[M_{d}^{\prime}(d)-M_{d}^{\prime}(-d)\right]=b \int_{-d}^{d}\left(M_{d}-M_{0}\right)(\xi) d \xi$.

By Propositions 3.2 and 3.3 and Lemmas 4.1 and 4.2, in the limit $d \rightarrow \infty$,

$$
Y\left(c R_{f}-v\right) S^{+}=b \lim _{d \rightarrow \infty} \int_{-d}^{d}\left(M_{d}-M_{0}\right)(\xi) d \xi=b \int_{-\infty}^{\infty}\left(M-M_{0}\right)(\xi) d \xi
$$

Multiplying (4.1) by $Y$, adding it to (4.3), integrating the resulting equation from $\xi=-\infty$ to $\xi=+\infty$, and using boundary conditions as well as the decay of the limiting functions at infinities, we end up with

$$
Y\left(c R_{f}-v\right) \tilde{S}^{+}=b \int_{-\infty}^{\infty}\left(M-M_{0}\right)(\xi) d \xi .
$$


Combining (4.14)-(4.15), we find that $S^{+}=\tilde{S}^{+}$, and similarly $A_{-}=\tilde{A}_{-}$. Thus, we have a solution $(S, A, M)$ for the system (4.1)-(4.3) satisfying $S(\infty)=S^{+}, S(-\infty)=$ $0, A(\infty)=0$, and $A(-\infty)=A_{-}$. The fact that $M(\xi)>M_{0}$ follows from the maximum principle. The proof is complete.

5. Traveling waves on the real line. Through results of the last section, we have established the existence of the regularized solutions denoted by $\left(S_{\varepsilon}, A_{\varepsilon}, M_{\varepsilon}, c_{\varepsilon}\right)$ over the real line. We show that they converge to the desired traveling wave solutions as $\varepsilon \rightarrow 0$. We have found $c_{\varepsilon}=c$ independent of $\varepsilon$. However, we improve the previous bounds on $S_{\varepsilon}, A_{\varepsilon}, M_{\varepsilon}$ and their derivatives so that they are independent of $\varepsilon$. Moreover, we show that the boundary conditions are valid for limiting functions $(S, A, M)$ as $\xi \rightarrow \infty$. The first step is to establish an upper bound on $M_{\varepsilon}$ independent of $\varepsilon$.

Lemma 5.1. For $M_{\varepsilon}$ of the system (4.1)-(4.3), $M_{\varepsilon}$ satisfies

$$
M_{0}<M_{\varepsilon} \leq M_{0}+Y\left(R_{f}-\frac{v}{c}\right) S^{+} \quad \forall \xi \in R^{1} .
$$

Proof. As mentioned, $\lim _{\varepsilon \rightarrow 0} M_{\varepsilon}=M \not \equiv M_{0}$. By Proposition 2.1 and Theorem 4.1, we have that $M_{0}<M_{\varepsilon}$. Therefore, $\exists \xi_{1} \in(-\infty, \infty)$ such that $M_{\varepsilon}\left(\xi_{1}\right)=$ $\sup _{\xi \in R^{1}} M_{\varepsilon}(\xi)=\bar{M}_{\varepsilon}>M_{0}$. Multiplying $Y$ by (4.1), adding it to (4.3), and integrating from $\xi_{1}$ to $\infty$, we obtain

$$
-Y D S_{\varepsilon}^{\prime}\left(\xi_{1}\right)+Y\left(c R_{f}-v\right)\left(S^{+}-S_{\varepsilon}\left(\xi_{1}\right)\right)+c\left(M_{0}-\bar{M}_{\varepsilon}\right)=b \int_{\xi_{1}}^{\infty}\left(M_{\varepsilon}-M_{0}\right)(\xi) d \xi
$$

Note that $M_{\varepsilon}^{\prime}\left(\xi_{1}\right)=0$ and $\int_{\xi_{1}}^{+\infty}\left(M_{\varepsilon}-M_{0}\right) d \xi \geq 0$. Solving for $\bar{M}_{\varepsilon}$,

$$
\bar{M}_{\varepsilon} \leq M_{0}+Y\left(R_{f}-\frac{v}{c}\right) S^{+} .
$$

It is clear that $M_{0}<M_{\varepsilon}(\xi) \leq \bar{M}_{\varepsilon} \leq M_{0}+Y\left(R_{f}-v / c\right) S^{+}$. Substituting in the expression for $c$ in Proposition 3.4, we obtain the bound given in the main theorem. The proof of the lemma is complete.

Proof of the main theorem. First by Lemma 5.1, $\left(S_{\varepsilon}, A_{\varepsilon}, M_{\varepsilon}\right) \rightarrow(S, A, M)$ in $C_{l o c}^{1}$. We want to show that $S$ decays to zero as $\xi \rightarrow-\infty$ (as we showed previously in Corollary 3.2). We see that the upper bound for $S_{\varepsilon}$ on $\xi \leq 0$ depends on $r_{2}$ which in turn depends on $\eta_{2}(d, \varepsilon)$. We recall from Proposition 3.2 that $\eta_{2}$ is a lower bound for $R_{s} / S_{\varepsilon}$. Therefore, we can improve this lower bound by substituting in the upper bound for $M_{\varepsilon}$ as given in Lemma 5.1. Then we have for $\varepsilon \in[0,1]$,

$$
\eta=\frac{M_{0} \theta}{\left(1+M_{0}+Y\left(R_{f}-\frac{v}{c}\right) S^{+}\right)\left(1+S^{+}\right)\left(1+A_{-}\right)} \leq \eta_{2}^{\varepsilon} \leq \frac{R_{s}^{\varepsilon}}{S_{\varepsilon}} .
$$

We establish an upper bound for $S_{\varepsilon}$ on the negative real line by following the proof of Corollary 3.2. In this way, we find a bound which is independent of $\varepsilon$ and as such, $S_{\varepsilon} \rightarrow S$ and in the limit $\xi \rightarrow-\infty$ decays to zero.

We must also establish that $\liminf _{\varepsilon \rightarrow 0} S_{\varepsilon}(0)>0$ so that the derivatives for $S, A$ tend to zero at infinity. Following the proof of Lemma 3.3, we can derive the analogous inequality

$$
0 \geq S^{+}-\frac{\theta_{0} \delta_{2}}{\gamma \delta_{1}}
$$


Since all these constants are independent of $\varepsilon, \theta_{0}$ can be chosen small enough so that the right-hand side is positive without being dependent on $\varepsilon$. Thus the bound holds, and so $S(0)>0$.

As such, it is easy to deduce that the second part of Corollary 3.2 can be reproduced independent of $\varepsilon$. The first inequality of Corollary 3.2 follows with $\beta_{1}$ independent of $\varepsilon$. Given the results of Lemma 5.1, and because $S(0)>0$, we have that $\eta_{1}>0$ independent of $\varepsilon$. Therefore, $\lim _{\xi \rightarrow-\infty} S=0$. Similarly, as $\xi \rightarrow \infty, A$ decays to zero.

Next, we reproduce Lemma 4.1 to find the decay properties of $M$ near infinity. We begin by improving the upper bound of $R_{s}^{\varepsilon}$ in Lemma 4.1. We see that by Lemma 5.1 ,

$$
R_{s}^{\varepsilon} \leq\left(M_{0}+Y\left(R_{f}-\frac{v}{c}\right) S^{+}\right) \theta e^{-\beta_{1} \xi}
$$

We construct an upper solution $\bar{m}_{\varepsilon}$ to $m_{\varepsilon}=M_{\varepsilon}-M_{0}$ on $\left[\xi_{1}, \infty\right]$ which solves

$$
\begin{aligned}
& \varepsilon \bar{m}_{\varepsilon}^{\prime \prime}+c \bar{m}_{\varepsilon}^{\prime}-b \bar{m}_{\varepsilon}=-Y\left(M_{0}+Y\left(R_{f}-\frac{v}{c}\right) S^{+}\right) \theta e^{-\beta_{1} \xi}, \\
& \bar{m}_{\varepsilon}\left(\xi_{1}\right) \geq m_{\varepsilon}\left(\xi_{1}\right)>0 .
\end{aligned}
$$

The solution to (5.6) is

$$
\bar{m}_{\varepsilon}=-y_{1} \frac{Y\left(M_{0}+Y\left(R_{f}-\frac{v}{c}\right) S^{+}\right) \theta e^{-\beta_{1} \xi}}{\varepsilon \beta_{1}^{2}-c \beta_{1}-b},
$$

where $0<\beta_{1} \leq \frac{c-v+\sqrt{(v-c)^{2}+4 D \eta_{1}}}{2 D}$ and $y_{1}$ is a positive constant chosen so that the inequality in (5.6) holds at $\xi_{1}$. In the limit as $\varepsilon \rightarrow 0$,

$$
\bar{m}_{\varepsilon} \rightarrow y_{1} \frac{Y\left(M_{0}+Y\left(R_{f}-\frac{v}{c}\right) S^{+}\right) \theta e^{-\beta_{1} \xi}}{\left(c \beta_{1}+b\right)} \equiv \bar{m}_{0} .
$$

Thus, in the limit $\xi \rightarrow+\infty$, we have that $\bar{m}_{0} \rightarrow 0$, which implies that $m(\xi) \rightarrow 0$ as $\xi \rightarrow+\infty$ or $\lim _{\xi \rightarrow+\infty} M(\xi)=M_{0}$. Similarly, $\lim _{\xi \rightarrow-\infty} M(\xi)=M_{0}$.

Justifying the limits $\lim _{\xi \rightarrow-\infty} A=A_{-}$and $\lim _{\xi \rightarrow+\infty} S=S^{+}$as in the proof of Theorem 4.1, we have shown that the limiting functions $(S, A, M, c)$ are traveling wave solutions satisfying all the boundary conditions.

Finally, we establish the strict inequalities for the wave profiles. By the strong elliptic maximum principle, $0<S(\xi)<S^{+}$, and $0<A(\xi)<A_{-}$for any finite $\xi$. If $M\left(\xi_{1}\right)=M_{0}$, for a finite $\xi_{1}$, then $M^{\prime}\left(\xi_{1}\right)=0$. The $M$ equation evaluated at $\xi=\xi_{1}$ cannot hold thanks to $S\left(\xi_{1}\right) A\left(\xi_{1}\right)>0$. Hence, $M(\xi)>M_{0}$ for any finite $\xi$.

If $A^{\prime}\left(\xi_{2}\right)=0$ for a finite $\xi_{2}$, then by the $A$ equation evaluated at $\xi=\xi_{2}$, we see that $A^{\prime \prime}\left(\xi_{2}\right)=\gamma R_{s}\left(\xi_{2}\right)>0$. It follows that $\xi_{2}$ is a local minimal point, which contradicts the fact that $A$ is monotone decreasing. Hence, $A^{\prime}<0$ for any finite $\xi$. Similarly, $S^{\prime}>0$, for any finite $\xi$. The proof of the main theorem is complete.

6. Existence of traveling waves in the zero-diffusion model. In this section, we prove Theorem 1.2. We have already shown that traveling wave solutions exist to (1.1)-(1.3) for $D>0$. For the case when $D=0$, the equations are reduced to first order. Using the conserved quantity, $\gamma R_{f} S-A$, and defining the new variables, $u=\gamma R_{f} S-A$ and $w=\gamma S-A,(1.1)-(1.3)$ are transformed into conservative form

$$
u_{t}+v w_{x}=0
$$




$$
\begin{aligned}
w_{t}+v / R_{f}((R+1) w-u)_{x} & =\varepsilon\left(u-R_{f} w\right)(u-w) / G, \\
M_{t}-b\left(M-M_{0}\right) & =\frac{Y M\left(u-R_{f} w\right)(u-w)}{\gamma(R-1)^{2} K_{S} K_{A} G},
\end{aligned}
$$

where $\varepsilon=R_{f}^{-1}\left(R_{f}-1\right)^{-1}\left(K_{A} K_{S}\right)^{-1}$, and $G(A, S)=\left(1+K_{A}^{-1} A\right)\left(1+K_{S}^{-1} S\right)$. When rewritten in the traveling wave variable, $\xi$, (6.1) becomes $u_{\xi}=(v / c) w_{\xi}$. This relationship restricts the flow in the phase space to two-dimensional planes. The two-by-two dynamical system is then

$$
\begin{aligned}
w_{\xi} & =-\frac{R_{f} \varepsilon\left(A_{-}+\gamma R_{f} S^{+}\right)(w-\gamma S)\left(w+A_{-}\right) M}{v\left(A_{-}+\gamma S^{+}\right) G} \\
M_{\xi} & =\frac{b\left(A_{-}+\gamma R_{f} S^{+}\right)\left(M-M_{0}\right)}{v\left(A_{-}+\gamma S^{+}\right)}+\frac{Y A_{-} S^{+}\left(A_{-}+\gamma R_{f} S^{+}\right)(w-\gamma S)\left(w+A_{-}\right) M}{v\left(A_{-}+\gamma S^{+}\right)^{3} K_{S} K_{A} G} .
\end{aligned}
$$

There are two equilibrium points in the phase plane $(w, M):\left(\gamma S^{+}, M_{0}\right)$ and $\left(-A_{-}, M_{0}\right)$. The eigenvalues governing the flow near $\left(\gamma S^{+}, M_{0}\right)$ are

$$
\lambda_{1}=\frac{b\left(A_{-}+\gamma R_{f} S^{+}\right)}{v\left(A_{-}+\gamma S^{+}\right)}>0 \quad \text { and } \quad \lambda_{2}=\frac{-R_{f} \varepsilon\left(A_{-}+\gamma R_{f} S^{+}\right)}{v}<0 .
$$

The eigenvalues for the flow near $\left(-A_{-}, M_{0}\right)$ are both positive. Thus, $\left(\gamma S^{+}, M_{0}\right)$ is a saddle point and $\left(-A_{-}, M_{0}\right)$ is an unstable node. In order to have a traveling wave solution, at least one of the unstable manifolds emanating from the point $\left(-A_{-}, M_{0}\right)$ must intersect the two-dimensional stable manifold of $\left(\gamma S^{+}, M_{0}\right)$. It is straightforward to show that there is a unique path between the two equilibrium points. Thus, the proof of Theorem 1.2 is complete.

Acknowledgments. The authors would like to thank A. Valocchi and S. Oya for communicating their work in progress and the preprint of their paper [8]. J. X. Xin would like to thank Y. Oono for kindly introducing him to the biodegradation model studied in this paper. Both authors wish to thank J. Hyman and B. Travis of the Los Alamos National Laboratory for their interest in this work. The authors also wish to thank M. Brusseau and L. Xie for their constructive comments.

\section{REFERENCES}

[1] H. Berestycki, B. Nicolaenko, And B. Sheurer, Traveling wave solutions to combustion models and their singular limits, SIAM J. Math. Anal., 16 (1985), pp. 1207-1242.

[2] R. Borden And P. Bedient, Transport of dissolved hydrocarbons influenced by oxygen-limited biodegradation, Water Resources Res., 22 (1986), pp. 1973-1982.

[3] C. Chiang, C. Dawson, and M. Wheeler, Modeling of in-situ bioremediation of organic compounds in groundwater, Transport in Porous Media, 6 (1991), pp. 667-702.

[4] D. Gilbarg and N. Trudinger, Elliptic Partial Differential Equations of Second Order, 2nd edition, Springer-Verlag, New York, 1983.

[5] F. J. Molz, M. A. Widdowson, And L. D. Benefield, Simulation of microbial growth dynamics coupled to nutrient and oxygen transport in porous media, Water Resources Res., 22 (1986), pp. 1207-1216.

[6] National Research Council, In Situ Bioremediation, When Does It Work?, Water Science and Technology Board, National Academy Press, Washington, D.C., 1993.

[7] J. Odencrantz, A. Valocchi, And B. Rittman, Modeling the interaction of sorption and biodegradation on transport in ground water in situ bioremediation systems, in Proceedings of the 1993 Ground Water Modeling Conference, E. Poeter, S. Ashlock, and J. Proud, eds., International Ground Water Modeling Center, Golden, CO, 1993, pp. 2-3-2-12. 
[8] S. OYA AND A. VALOCCHI, Characterization of traveling waves and analytical estimation of pollutant removal in one-dimensional subsurface bioremediation modeling, Water Resources Res., 33 (1997), pp. 1117-1127.

[9] M. Protter and H. Weinberger, Maximum Principles in Differential Equations, PrenticeHall, Englewood Cliffs, NJ, 1967.

[10] A. VAlocchI, Traveling Wave Behavior During Subsurface Transport of Biologically Reactive Contaminants: Implications for In Situ Bioremediation, private communication, Dept. of Civil Engrg., Univ. of Illinois, Urbana, IL, 1995.

[11] X. XIN, Existence and uniqueness of traveling waves in a reaction-diffusion equation with combustion nonlinearity, Indiana Univ. Math J., 40 (1991), pp. 985-1008.

[12] J. X. XIN, Existence of multidimensional traveling waves in transport of reactive solutes through periodic porous media, Arch. Rat. Mech. Anal., 128 (1994), pp. 75-103.

[13] E. ZeIdLer, Nonlinear Functional Analysis, Springer-Verlag, New York, 1984. 